\title{
A Corey-Seebach Macrocyclisation Strategy for the Synthesis of Riccardin C and an Unnatural Macrocyclic Bisbibenzyl Analogue
}

\author{
Faisal A. Almalki ${ }^{[a]}$ and David C. Harrowven*[a]
}

\begin{abstract}
A total synthesis of riccardin $\mathrm{C}$ has been accomplished using a Corey-Seebach reaction to effect macrocyclisation. The versatility of the
\end{abstract}

strategy has also been demonstrated with a mimetic synthesis of an unnatural bisbibenzyl analogue.
Keywords: natural products $\bullet$ total synthesis $\bullet$ macrocyclisation • synthesis (org.) • Corey-Seebach

\section{Introduction}

Macrocyclic bisbibenzyl natural products have largely been found in liverworts and other bryophytes, ${ }^{[1,2]}$ with riccardin C $\mathbf{1 0}$ alone in being identified in a higher plant species. ${ }^{[3,4]}$ In Nature, all are derived from lunularin 4 (Figure 1)..$^{[5]}$ First, an oxidative dimerisation leads to perrottetin E $\mathbf{3}$ or isoperrottetin A $\mathbf{6}$. Then, related oxidative biaryl or biaryl ether formation occurs leading to the macrocyclic cores $\mathbf{1}, \mathbf{2}, \mathbf{7}$ and $\mathbf{8}$ of the different familial groups. ${ }^{[1,2,5]}$ Siblings are then derived by benzylic or arene oxidation, phenolic methylation, oxidative dimerisation or further oxidative cyclisation (e.g. $10 \rightarrow$ cavicularin 9 and asterelin A 11, ${ }^{[6,7]}$ Figure 2).

The largest familial group is based on macrocyclic core $\mathbf{2}$ and has riccardin $\mathrm{C} \mathbf{1 0}$ at its helm (Figures 1 and 2). ${ }^{[3,4]}$ A popular synthetic target for many years, ${ }^{[8-14]}$ riccardin $\mathrm{C}$ recently gained prominence when a screen seeking new leads against cardiovascular disease found that it acted as an agonist for the cholesterol-regulating liver $\mathrm{X}$ receptor LXR $\alpha$ and an antagonist for LXR $\beta .^{[11]}$ Indeed, it has been tested in many pharmacological screens, ${ }^{[1,2]}$ to reveal antifungal, ${ }^{[15]}$ antibacterial, ${ }^{[16]}$ and cytotoxic activity against various cancer cell lines. ${ }^{[17]}$ A limited number of structure-activity relationships have also been conducted, largely based on isolated natural products and simple analogues derived therefrom. ${ }^{[11,18]}$

Herein, we show how convergent synthesis can unlock pathways to analogues with and without precedent in Nature, as demonstrated by syntheses of riccardin $\mathrm{C}$ and the unnatural analogue 5. Although the

[a] F. A. Almalki and Prof. D. C. Harrowven

Chemistry, University of Southampton, Highfield, Southampton Hampshire, SO17 1BJ, UK.

Fax: (+) 44 (0)23 80596805

E-mail: dch2@soton.ac.uk

Supporting information for this article is available on the WWW under ((will be filled in by the editorial staff)). ring system in $\mathbf{5}$ has a similar constitution to those found in Nature, the biaryl bond linking arenes B and D is not consistent with macrocyclic bisbibenzyl biosynthesis as in natural products these rings are necessarily bound within the macrocycle via ortho- and para-linkages. ${ }^{[1,2,5]}$ Through the simple expedient of activating either the ortho- or the meta-carbon in arene $\mathrm{D}$, we were able to gain access to both a natural and an unnatural product series respectively (Schemes 1 and 2).

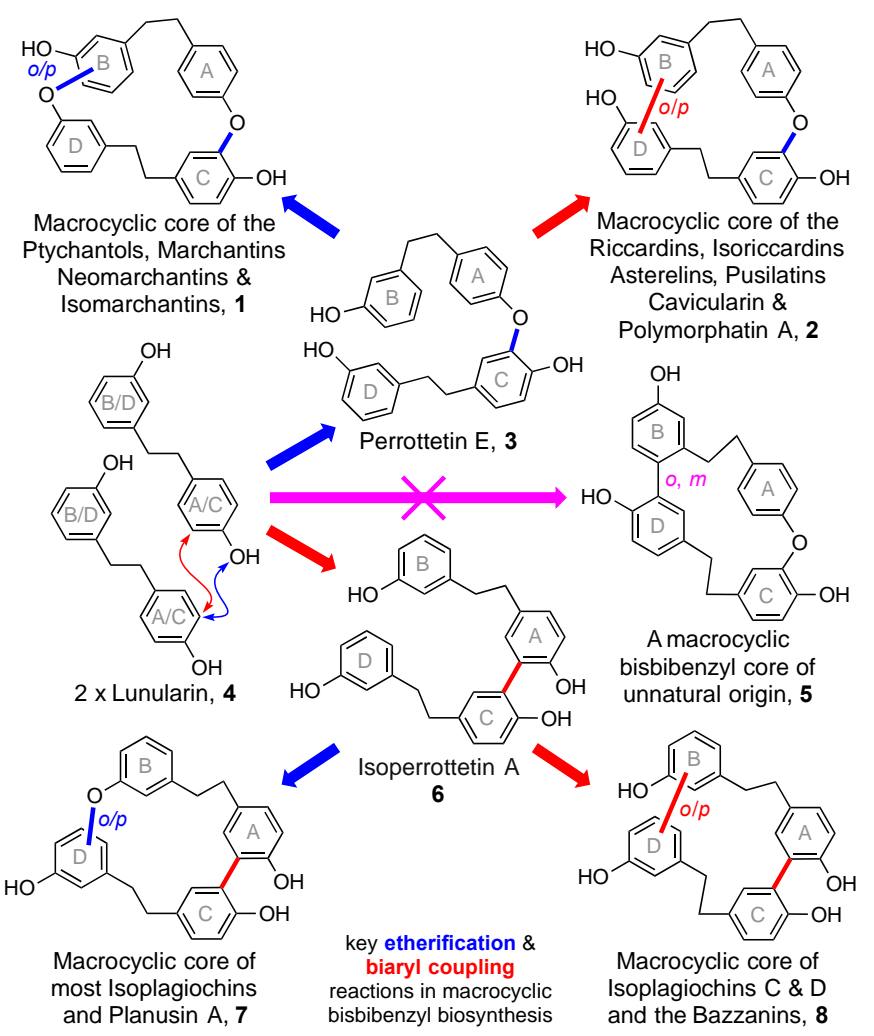

Figure 1. Structural relationships in macrocyclic bisbibenzyl natural products and the unnatural analogue herein targeted by synthesis. 
Mindful of the fact that syntheses of riccardin C $\mathbf{1 0}$ proceeding via macrocycle $\mathbf{1 2}$ also constitute formal total syntheses of cavicularin $\mathbf{9}^{[10,12,13,19,20]}$ and asterelin A 11 (Figure 2), ${ }^{[21]}$ we decided to deploy an orthogonal protecting group strategy for the phenol in arene D to those in arenes B and C. In previous syntheses several strategies have been developed to tackle the challenge of forming its 18 membered ring (Figure 1). ${ }^{[8-14,20]}$ These include macrocyclisation protocols using Wurtz, ${ }^{[8]}$ Wittig $^{[9-11]}$ and McMurry ${ }^{[11,12]}$ reactions to effect closure via either of the ethano bridges; $\operatorname{Pd}(0)$ crosscoupling ${ }^{[13]}$ and $\mathrm{S}_{\mathrm{N}} \mathrm{Ar}^{[14]}$ reactions for closure via the biaryl or biaryl ether linkages and two approaches to cavicularin based on the de novo construction of a arene A. ${ }^{[20]}$ Herein we report a new strategy wherein a Corey-Seebach reaction ${ }^{[22-24]}$ is used to achieve the critical macrocyclisation step. ${ }^{[25]}$ Importantly, while this reaction has been widely use in macrocycle synthesis, ${ }^{[22-24]}$ its use in the context of macrocyclisation is extremely rare. ${ }^{[25]}$

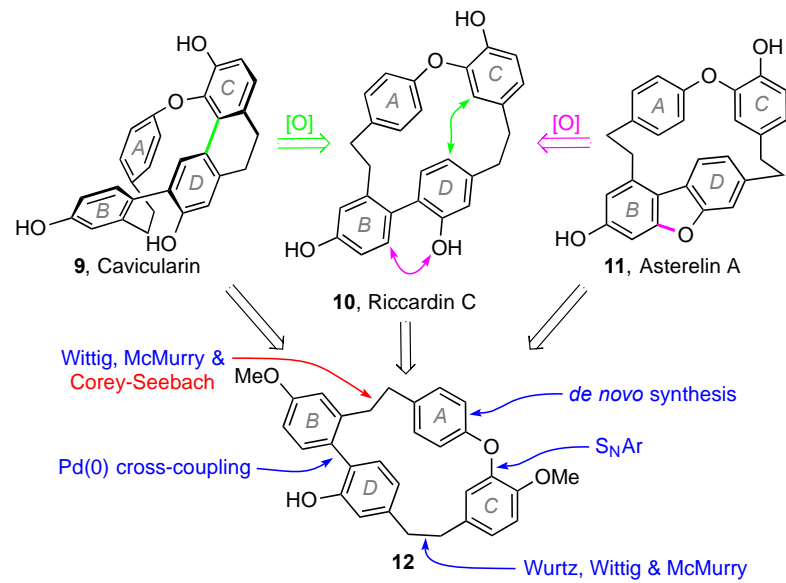

Figure 2. Macrocyclisation strategies used to prepare riccardin C (blue), the approach detailed herein (red) and formal syntheses by intersection with $\mathbf{1 2}$.

\section{Results and Discussion}

\section{Development of a Convergent Synthesis.}

Drawing on previous experience, ${ }^{[10,12]}$ we identified arenes 16-19 as the key building blocks required for our synthesis, as all but arene 17 were products of commerce (Scheme 1). Moreover, the synthesis of $\mathbf{1 7}$ from catechol $\mathbf{1 3}$ was readily achieved by sequential benzylation, triflation and Wittig methylenation using standard protocols. In the first step we found that 2 equivalents of $\mathrm{NaH}$ were needed to achieve optimal regioselectivity while in the third step its use prevented triflate hydrolysis, as observed when employing KOtBu. The stage was now set to assemble the macrocyclisation precursor 27. First, union of arenes D and B, $\mathbf{1 6}$ and $\mathbf{1 7}$, was accomplished by means of a Suzuki-Miyaura coupling ${ }^{[26]}$ then the resulting biaryl aldehyde 20 was reduced to benzyl alcohol 21. Contemporaneously, arenes $\mathrm{C}$ and $\mathrm{A}, \mathbf{1 8}$ and 19, were advanced to biaryl ether $\mathbf{2 3}$ using the known $\mathrm{S}_{\mathrm{N}} \mathrm{Ar}$ and iodination sequence. ${ }^{[12]}$ At this juncture various permutations were examined to advance the synthesis. The most convenient involved coupling of alcohol $\mathbf{2 1}$ and aldehyde $\mathbf{2 3}$ prior to installation of the dithiane unit (Scheme 1). In this way the Heck reaction leading to tetraarene $\mathbf{2 5}$ could be realised in a good yield consistently using catalytic $\mathrm{Pd}(\mathrm{OAc})_{2}$ under ligand-free conditions. ${ }^{[27]}$ Indeed, the yield given was almost twice that attained when the order of these steps was reversed. Alkene reduction to $\mathbf{2 6}$ was next facilitated by diimide, ${ }^{[10,12]}$ then the alcohol was transformed into chloride 27 through the action of $\mathrm{MsCl}$ or $\mathrm{SOCl}_{2}$
(Scheme 2). Though the former proved capricious when using 2,6lutidine as base, a good yield was attained using DBU with $\mathrm{LiCl}^{[28]}$

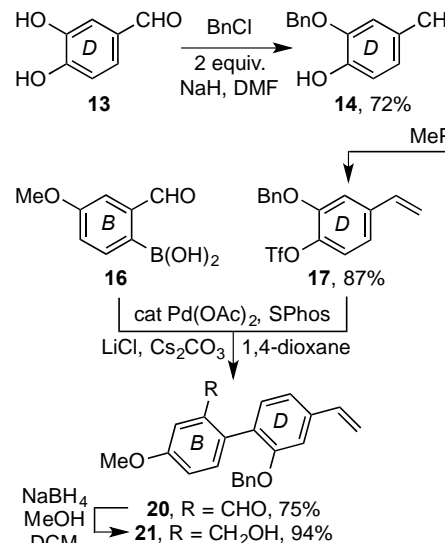

$\mathrm{MeOH} \longrightarrow 21, \mathrm{R}=\mathrm{CH}_{2} \mathrm{OH}, 94 \%$

cat $\mathrm{Pd}(\mathrm{OAc})_{2}, \mathrm{~K}_{3} \mathrm{PO}_{4}$, DMA

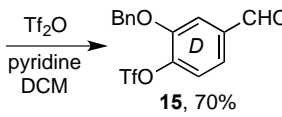

$\mathrm{MePPh}_{3} \mathrm{Br}, \mathrm{NaH}, \mathrm{THF}$

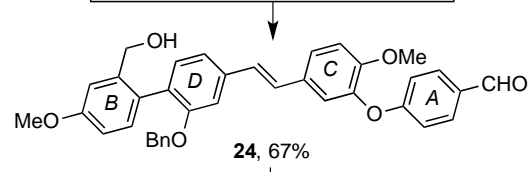

24, $67 \%$

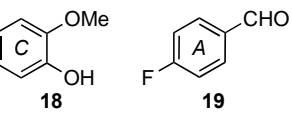

$18 \quad 19$

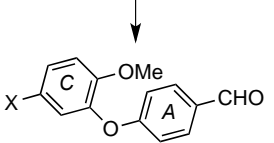

22, $\mathrm{X}=\mathrm{H}, 96 \%$ NIS, TFA 23, $X=I, 95 \% \varangle M e C N$
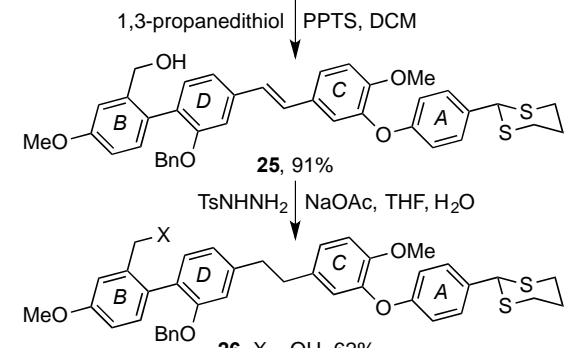

$\left.\mathrm{SOCl}_{2}, \mathrm{py}, \mathrm{DCM} \longrightarrow 27, \mathrm{X}=\mathrm{Cl}, 67 \%[63 \%] \div\right][\mathrm{MsCl}, \mathrm{DBU}, \mathrm{LiCl}, \mathrm{DCM}]$

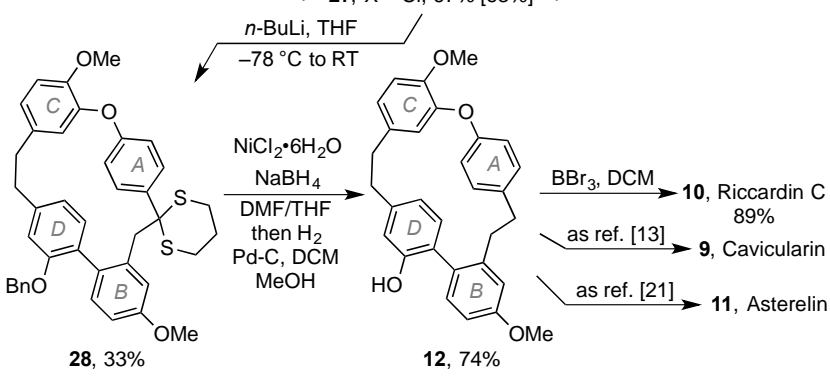

Scheme 1. Total synthesis of riccardin $\mathrm{C}$ and formal total syntheses of cavicularin and asterelin A.

The stage was now set to examine the macrocyclisation step. ${ }^{[25]}$ Pleasingly, deprotonation of dithiane 27 with $\mathrm{BuLi}$ at $-78{ }^{\circ} \mathrm{C}$, followed by warming to ambient temperature, gave the requisite macrocycle $\mathbf{2 8}$ in $33 \%$ yield. Reductive removal of the dithiane ${ }^{[29]}$ and benzyl ether functions then gave riccardin $\mathrm{C}$ dimethyl ether $\mathbf{1 2}$ from which riccardin C $\mathbf{1 0}$ was readily prepared by deprotection with boron tribromide. As routes to cavicularin $\mathbf{9}^{[19]}$ and asterelin A $\mathbf{1 1}^{[22]}$ have been described from 12, the approach also constitutes formal total syntheses of those natural products.

Having demonstrated the applicability of the convergent 'plug and play' strategy in natural product target synthesis, we next sought to show how it could be adapted to give access to an unnatural macrocyclic bisbibenzyl motif such as $\mathbf{5}$. To that end, we re-ran the entire synthesis using the regioisomeric styrene $\mathbf{3 1}$ in place of the arene D component 17 (Scheme 2). Pleasingly, we were able to mirror every step in the synthesis and achieved an improved outcome for the Corey-Seebach marcocyclisation, $\mathbf{3 7} \rightarrow \mathbf{3 8}$, which was realised in $48 \%$ yield. 

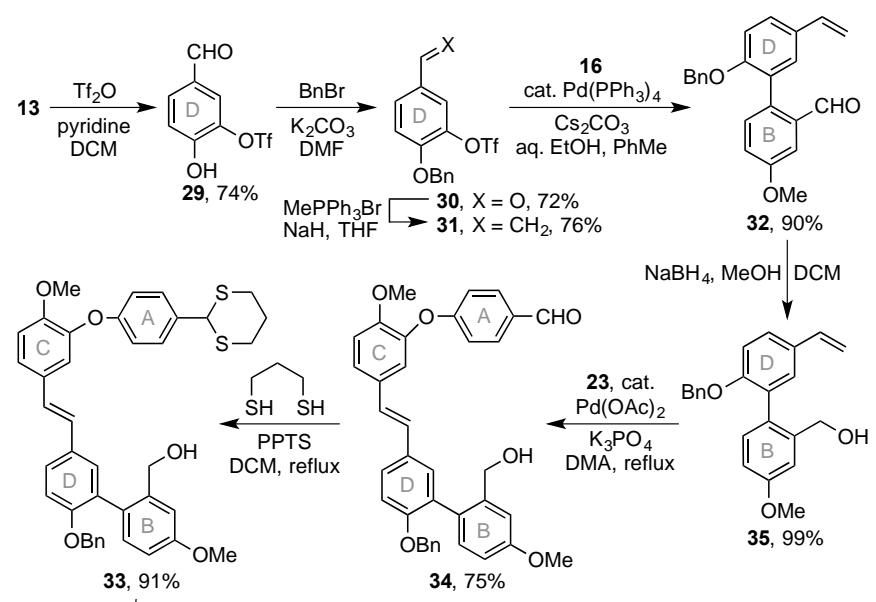

$\mathrm{TsNHNH}_{2} / \mathrm{NaOAc}$, aq. THF, reflux

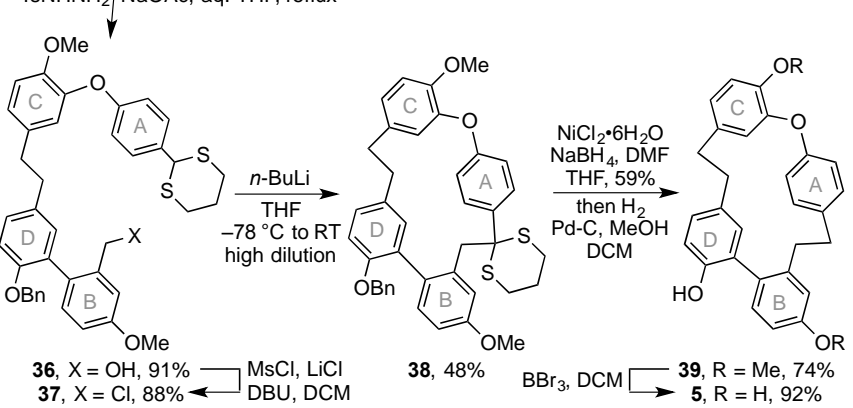

Scheme 2. A re-run of the synthesis with regioisomeric styrene 32 to prepare the unnatural macrocyclic bisbibenzyl analogue $\mathbf{5}$.

\section{Conclusion}

In summary, a total synthesis of riccardin $\mathbf{C ~} \mathbf{1 0}$ has been developed in which macrocyclisation was achieved by means of a CoreySeebach reaction (Scheme 1). Importantly, while that reaction is synonymous with the assembly of macrocyclic products, ${ }^{[23,24]}$ its use in the context of macrocyclic ring closure is extremely rare. ${ }^{[25]} \mathrm{We}$ have also shown how the convergent nature of our synthesis allows it to be used as a template to build related macrocyclic bisbibenzyls, as exemplified by our mimetic synthesis of the unnatural analogue 5 (Scheme 2).

\section{Experimental Section}

Melting points were recorded on a Reichert (Austria) apparatus and are uncorrected Infrared spectra were recorded neat as thin films or as solid compressions using the Golden Gate ATR method. ${ }^{1} \mathrm{H}$ NMR and ${ }^{13} \mathrm{C}$ NMR spectra were recorded on a Bruke DPX400 (400/100 MHz) spectrometer at $298 \mathrm{~K}$. Experiments were carried out in $\mathrm{CDCl} 3$, unless otherwise stated, that had been stored over dried $\mathrm{K}_{2} \mathrm{CO}_{3}$ to neutralise trace acidity. High resolution mass spectrometry was carried out using a MaXis (Bruke Daltonics, Bremen, Germany) mass spectrometer equipped with a time of flight analyser or a Thermo Mat 900 XP double focusing high resolution mass spectrometer All samples were analyzed and recorded by Ms. Julie Herniman at the University of Southampton. Low resolution mass spectrometry was carried out using electrospray ionisation on a directly injected WATERS quadrupole MSD using ESI+ with $\mathrm{MeOH} /$ acetonitrile as solvent. X- ray data were recorded using a Rigaku AFC12 FRE$\mathrm{HF}$ diffractometer equipped with an Oxford Cryosystems low-temperature apparatus operating at $100 \mathrm{~K}$ [CCDC number for compound 5 is 1438902].

2-(Benzyloxy)-4-formylphenyl trifluoromethanesulfonate (15): To a cooled $\left(0{ }^{\circ} \mathrm{C}\right)$ solution of phenol $1 \mathbf{1}^{[30]}(110 \mathrm{mg}, 0.48 \mathrm{mmol})$ and pyridine $(0.046 \mathrm{~mL}, 0.57 \mathrm{mmol})$ in DCM $(2 \mathrm{~mL})$ was added $\mathrm{Tf}_{2} \mathrm{O}(0.088 \mathrm{~mL}, 0.52 \mathrm{mmol})$. After $5 \mathrm{~h}$ at RT the reaction mixture was concentrated in vacuo and purified by column chromatography (20\% $\mathrm{Et}_{2} \mathrm{O} /$ petrol) to afford the title compound 15 as a colourless oil $(120 \mathrm{mg}, 70 \%) ;{ }^{1} \mathrm{H}$ NMR $\left(400 \mathrm{MHz}, \mathrm{CDCl}_{3}\right): \delta=9.97(\mathrm{~s}, 1 \mathrm{H}), 7.64(\mathrm{~d}, J=1.7 \mathrm{~Hz}, 1 \mathrm{H}), 7.53(\mathrm{dd}, J=8.1,1.8 \mathrm{~Hz}$ $1 \mathrm{H}), 7.51-7.47(\mathrm{~m}, 2 \mathrm{H}), 7.44(\mathrm{~d}, J=8.2 \mathrm{~Hz}, 1 \mathrm{H}), 7.46-7.35(\mathrm{~m}, 3 \mathrm{H}), 5.26(\mathrm{~s}, 2 \mathrm{H}) \mathrm{ppm}$ ${ }^{13} \mathrm{C}$ NMR $\left(100 \mathrm{MHz}, \mathrm{CDCl}_{3}\right): \delta=190.2(\mathrm{CH}), 151.3(\mathrm{C}), 142.8(\mathrm{C}), 136.7(\mathrm{C}), 134.8$ $(\mathrm{C}), 128.7(2 \times \mathrm{CH}), 128.5(\mathrm{CH}), 127.4(2 \times \mathrm{CH}), 124.1(\mathrm{CH}), 123.2(\mathrm{CH}), 118.6(\mathrm{q}, J=$ $\left.320.6 \mathrm{~Hz}, \mathrm{CF}_{3}\right), 113.1(\mathrm{CH}), 71.4\left(\mathrm{CH}_{2}\right) \mathrm{ppm} ;{ }^{19} \mathrm{~F} \mathrm{NMR}\left(376 \mathrm{MHz}, \mathrm{CDCl}_{3}\right): \delta=-73.83$ ppm; IR (film): v 2838, 1701, 1602, 1497, 1423, 1381, 1205, 1136, 1100, 1007, 871 ,
$736 \mathrm{~cm}^{-1}$; MS (EI') m/z (\%): $360\left(\mathrm{M}^{+}, 2 \%\right), 108(5 \%), 108(30 \%), 91$ (100\%); HRMS (EI): $m / z$ calcd for $\mathrm{C}_{15} \mathrm{H}_{11} \mathrm{O}_{5} \mathrm{~F}_{3} \mathrm{~S}[\mathrm{M}]^{+} 360.02738$; found: 360.02703 .

2-(Benzyloxy)-4-vinylphenyl trifluoromethanesulfonate (17): To a cooled $\left(0{ }^{\circ} \mathrm{C}\right)$ solution of aldehyde $15(8.25 \mathrm{~g}, 22.89 \mathrm{mmol})$ in THF $(230 \mathrm{~mL})$ were added sequentially $60 \%$ sodium hydride in mineral oil $(2.75 \mathrm{~g}, 68.69 \mathrm{mmol})$ and methyltriphenylphosphonium bromide $(12.26 \mathrm{~g}, 34.33 \mathrm{mmol})$. After $18 \mathrm{~h}$ at RT the reaction mixture was concentrated in vacuo and purified by flash column chromatography $\left(10 \% \mathrm{Et}_{2} \mathrm{O} /\right.$ petrol $)$ to afford the title compound 17 as a colourless oil $(7.20 \mathrm{~g}, 87 \%) ;{ }^{1} \mathrm{H} \mathrm{NMR}(400 \mathrm{MHz}$, $\left.\mathrm{CDCl}_{3}\right): \delta=7.51(\mathrm{br} \mathrm{d}, J=8.7 \mathrm{~Hz}, 2 \mathrm{H}), 7.46-7.34(\mathrm{~m}, 3 \mathrm{H}), 7.22(\mathrm{~d}, J=8.3 \mathrm{~Hz}, 1 \mathrm{H})$, $7.13(\mathrm{~d}, J=2.0 \mathrm{~Hz}, 1 \mathrm{H}), 7.04(\mathrm{dd}, J=8.4,1.8 \mathrm{~Hz}, 1 \mathrm{H}), 6.68(\mathrm{dd}, J=17.5,10.9 \mathrm{~Hz}$ $1 \mathrm{H}), 5.74(\mathrm{~d}, J=17.5 \mathrm{~Hz}, 1 \mathrm{H}), 5.35(\mathrm{~d}, J=10.9 \mathrm{~Hz}, 1 \mathrm{H}), 5.21(\mathrm{~s}, 2 \mathrm{H}) \mathrm{ppm} ;{ }^{13} \mathrm{C}$ NMR $\left(100 \mathrm{MHz}, \mathrm{CDCl}_{3}\right): \delta=150.5(\mathrm{C}), 138.9(\mathrm{C}), 138.3(\mathrm{C}), 135.6(\mathrm{C}), 135.4(\mathrm{CH}), 128.6$ $(2 \times \mathrm{CH}), 128.2(\mathrm{CH}), 127.3(2 \times \mathrm{CH}), 122.4(\mathrm{CH}), 119.1(\mathrm{CH}), 115.9\left(\mathrm{CH}_{2}\right), 118.7(\mathrm{q}$, $\left.J=320.6 \mathrm{~Hz}, \mathrm{CF}_{3}\right), 111.9(\mathrm{CH}), 71.0\left(\mathrm{CH}_{2}\right) \mathrm{ppm} ;{ }^{19} \mathrm{~F} \mathrm{NMR}\left(376 \mathrm{MHz}, \mathrm{CDCl}_{3}\right): \delta=$ 73.95 ppm; IR (film): v 3035, 1596, 1502, 1418, 1275, 1247, 1203, 1179, 1137, 1101, 1010, $869 \mathrm{~cm}^{-1}$; MS $\left(\mathrm{EI}^{+}\right) \mathrm{m} / \mathrm{z}(\%): 358\left(\mathrm{M}^{+}, 12 \%\right), 91(100 \%)$; HRMS (EI): $\mathrm{m} / \mathrm{z}$ calcd for $\mathrm{C}_{16} \mathrm{H}_{13} \mathrm{O}_{4} \mathrm{~F}_{3} \mathrm{~S}$ [M] ${ }^{+}$358.04812; found: 358.04732 .

2'-(Benzyloxy)-4-methoxy-4'-vinyl-[1,1'-biphenyl]-2-carbaldehyde (20): To a solution of triflate $17(100 \mathrm{mg}, 0.27 \mathrm{mmol})$ and boronic acid $16(98.9 \mathrm{mg}, 0.55 \mathrm{mmol})$ in 1,4 dioxane $(2 \mathrm{~mL})$ was added $\mathrm{Cs}_{2} \mathrm{CO}_{3}(340 \mathrm{mg}, 1.06 \mathrm{mmol}), \mathrm{LiCl}(110 \mathrm{mg}, 2.67 \mathrm{mmol})$ and SPhos (43 mg, $0.1 \mathrm{mmol})$. The resulting mixture was degassed under argon for 15 min then $\mathrm{Pd}(\mathrm{OAc})_{2}(2.4 \mathrm{mg}, 0.01 \mathrm{mmol})$ was added. After heating at reflux for $3 \mathrm{~h}$, the reaction was cooled to $\mathrm{RT}$ and water $(5 \mathrm{~mL})$ added. The aqueous phase was extracted with EtOAc $(3 \times 10 \mathrm{~mL})$ then the combined organic phases were dried over $\mathrm{MgSO}_{4}$, concentrated in vacuo and purified by column chromatography $\left(10 \% \mathrm{Et}_{2} \mathrm{O} / \mathrm{petrol}\right)$ to afford the title compound $\mathbf{2 0}$ as a white solid (74 mg, 75\%); MP 76-77 ${ }^{\circ} \mathrm{C}$ $\left(\mathrm{Et}_{2} \mathrm{O} /\right.$ petrol$) ;{ }^{1} \mathrm{H}$ NMR $\left(400 \mathrm{MHz}, \mathrm{CDCl}_{3}\right): \delta=9.86(\mathrm{~s}, 1 \mathrm{H}), 7.52(\mathrm{~d}, J=2.8 \mathrm{~Hz}, 1 \mathrm{H})$, 7.34-7.31 (m, 2H), 7.30-7.28 (m, 2H), $7.25(\mathrm{~d}, J=7.8 \mathrm{~Hz}, 1 \mathrm{H}), 7.23-7.19(\mathrm{~m}, 3 \mathrm{H})$, $7.15(\mathrm{dd}, J=7.8,1.4 \mathrm{~Hz}, 1 \mathrm{H}), 7.10(\mathrm{~d}, J=1.3 \mathrm{~Hz}, 1 \mathrm{H}), 6.75(\mathrm{dd}, J=17.6,10.9 \mathrm{~Hz}$ $1 \mathrm{H}), 5.79(\mathrm{~d}, J=17.6 \mathrm{~Hz}, 1 \mathrm{H}), 5.33(\mathrm{~d}, J=10.9 \mathrm{~Hz}, 1 \mathrm{H}), 5.07(\mathrm{~s}, 2 \mathrm{H}), 3.92(\mathrm{~s}, 3 \mathrm{H})$ ppm; ${ }^{13} \mathrm{C}$ NMR (100 MHz, $\left.\mathrm{CDCl}_{3}\right): \delta=192.4(\mathrm{CH}), 159.2(\mathrm{C}), 156.1(\mathrm{C}), 139.2(\mathrm{C})$, $136.5(\mathrm{C}), 136.4(\mathrm{CH}), 135.0(\mathrm{C}), 134.6(\mathrm{C}), 132.5(\mathrm{CH}), 131.9(\mathrm{CH}), 128.5(2 \times \mathrm{CH})$, $127.8(\mathrm{CH}), 126.9(2 \times \mathrm{CH}), 126.9(\mathrm{C}), 121.1(\mathrm{CH}), 119.4(\mathrm{CH}), 114.7\left(\mathrm{CH}_{2}\right), 110.4$ $(\mathrm{CH}), 109.5(\mathrm{CH}), 70.6\left(\mathrm{CH}_{2}\right), 55.6\left(\mathrm{CH}_{3}\right) \mathrm{ppm}$; IR (solid): v 2932, 2851, 1687, 1603, $1486,1458,1416,1393,1316,1271,1241,1162,1136,1038 \mathrm{~cm}^{-1} ; \mathrm{MS}\left(\mathrm{EI}^{+}\right) \mathrm{m} / \mathrm{z}(\%)$ $344\left(\mathrm{M}^{+}, 3 \%\right), 237(35 \%), 91(100 \%)$; HRMS (EI): $\mathrm{m} / z$ calcd for $\mathrm{C}_{23} \mathrm{H}_{20} \mathrm{O}_{3}[\mathrm{M}]^{+}$ 344.14070; found: 344.14146

(2'-(Benzyloxy)-4-methoxy-4'-vinyl-[1,1'-biphenyl]-2-yl)methanol (21): To a suspension of aldehyde $20(1.69 \mathrm{~g}, 4.91 \mathrm{mmol})$ in methanol $(25 \mathrm{~mL})$ and $\mathrm{DCM}(25 \mathrm{~mL})$ at $0{ }^{\circ} \mathrm{C}$ was added $\mathrm{NaBH}_{4}(370 \mathrm{mg}, 9.82 \mathrm{mmol})$. The reaction mixture warmed to RT and after $4 \mathrm{~h}$, water $(20 \mathrm{~mL})$ was added. The aqueous phase was separated and extracted with $\mathrm{Et}_{2} \mathrm{O}$ (3 $\times 50 \mathrm{~mL}$ ) then the combined organic phases were dried over $\mathrm{MgSO}_{4}$, concentrated in vacuo and purified by column chromatography $\left(40 \% \mathrm{Et}_{2} \mathrm{O} /\right.$ petrol) to afford the title compound 21 as a colourless oil $(1.60 \mathrm{~g}, 94 \%) ;{ }^{1} \mathrm{H} \mathrm{NMR}\left(400 \mathrm{MHz}, \mathrm{CDCl}_{3}\right): \delta=7.37$ $7.29(\mathrm{~m}, 3 \mathrm{H}), 7.25-7.14(\mathrm{~m}, 7 \mathrm{H}), 6.96(\mathrm{dd}, J=8.3,2.7 \mathrm{~Hz}, 1 \mathrm{H}), 6.78(\mathrm{dd}, J=17.5,10.8$ $\mathrm{Hz}, 1 \mathrm{H}), 5.81(\mathrm{~d}, J=17.5 \mathrm{~Hz}, 1 \mathrm{H}), 5.34(\mathrm{~d}, J=10.9 \mathrm{~Hz}, 1 \mathrm{H}), 5.05(\mathrm{~s}, 2 \mathrm{H}), 4.47(\mathrm{br}$, $2 \mathrm{H}), 3.91(\mathrm{~s}, 3 \mathrm{H}), 2.24$ (br s, 1H) ppm; ${ }^{13} \mathrm{C}$ NMR $\left(100 \mathrm{MHz}, \mathrm{CDCl}_{3}\right): \delta=159.3(\mathrm{C})$ $155.9(\mathrm{C}), 140.8(\mathrm{C}), 138.3,(\mathrm{C}) 136.5(\mathrm{CH}), 136.4(\mathrm{C}), 131.7(\mathrm{CH}), 131.3(\mathrm{CH}), 130.3$ (C), $129.4(\mathrm{C}), 128.5(2 \times \mathrm{CH}), 127.9(\mathrm{CH}), 127.1(2 \times \mathrm{CH}), 119.8(\mathrm{CH}), 114.2\left(\mathrm{CH}_{2}\right)$, $113.4(\mathrm{CH}), 113.2(\mathrm{CH}), 111.5(\mathrm{CH}), 71.2\left(\mathrm{CH}_{2}\right), 63.6\left(\mathrm{CH}_{2}\right), 55.3\left(\mathrm{CH}_{3}\right) \mathrm{ppm}$; IR (film): v $3420 \mathrm{br}, 3008,2937,1604,1485,1271,1232,999,908,749 \mathrm{~cm}^{-1} ; \mathrm{MS}\left(\mathrm{EI}^{+}\right)$ $m / z(\%): 346\left(\mathrm{M}^{+}, 5 \%\right), 239(70 \%), 91(100 \%)$; HRMS (EI): $\mathrm{m} / \mathrm{z}$ calcd for $\mathrm{C}_{23} \mathrm{H}_{22} \mathrm{O}$ $[\mathrm{M}]^{+} 346.15635$; found: 346.15576 .

4-(5-Iodo-2-methoxyphenoxy)benzaldehyde (23): ${ }^{[10]}$ To a solution of benzaldehyde $\mathbf{2 2}^{[10,31]}(100 \mathrm{mg}, 0.44 \mathrm{mmol})$ in $\mathrm{MeCN}(3 \mathrm{~mL})$ were added trifluoroacetic acid $(0.009$ $\mathrm{mL}, 0.13 \mathrm{mmol}$ ) and $N$-iodosuccinimide $(110 \mathrm{mg}, 0.48 \mathrm{mmol})$. The reaction was heated at reflux for $13 \mathrm{~h}$ then cooled to RT and ice water $(2 \mathrm{~mL})$ was added. The product was extracted with $\mathrm{Et}_{2} \mathrm{O}(3 \times 5 \mathrm{~mL})$, then the combined organic phases were dried over $\mathrm{MgSO}_{4}$, concentrated in vacuo and purified by column chromatography (10-20\% $\mathrm{Et}_{2} \mathrm{O} /$ petrol) to afford the title compound $\mathbf{2 3}$ as an orange gum that crystallised on standing $(0.14 \mathrm{~g}, 90 \%)$; MP $48-49{ }^{\circ} \mathrm{C}\left(\mathrm{Et}_{2} \mathrm{O} /\right.$ petrol $)$ [Lit. $\left.48{ }^{\circ} \mathrm{C}\left(\mathrm{CH}_{2} \mathrm{Cl}_{2}\right)^{[100}\right]$; all spectroscopic and physical data matched that previously reported.

(E)-4-(5-(2-(2-(Benzyloxy)-2'-(hydroxymethyl)-4'-methoxy-[1, l'-biphenyl]-4-yl)vinyl)-2 methoxyphenoxy)benzaldehyde (24): A solution of styrene 21 (1.51 g, $4.36 \mathrm{mmol})$, iodide $23(1.40 \mathrm{~g}, 3.96 \mathrm{mmol})$ and potassium phosphate $(1.18 \mathrm{~g}, 5.54 \mathrm{mmol})$ in DMA (5 $\mathrm{mL}$ ) was degassed by sonication for 20 min under argon then $\mathrm{Pd}(\mathrm{OAc})_{2}(44 \mathrm{mg}, 0.19$ $\mathrm{mmol}$ ) was added. The reaction mixture was heated at $110{ }^{\circ} \mathrm{C}$ for $18 \mathrm{~h}$ then cooled to $\mathrm{RT}$, concentrated in vacuo and purified by column chromatography $\left(50 \% \mathrm{Et}_{2} \mathrm{O} /\right.$ petrol) to afford the title compound 24 as a white solid (1.53 g, 67\%) MP 66-67 ${ }^{\circ} \mathrm{C}$ (Et $2 \mathrm{O} /$ petrol); ${ }^{1} \mathrm{H}$ NMR $\left(400 \mathrm{MHz}, \mathrm{CDCl}_{3}\right): \delta=9.95(\mathrm{~s}, 1 \mathrm{H}), 7.88(\mathrm{~d}, J=8.8 \mathrm{~Hz}, 2 \mathrm{H})$, $7.40(\mathrm{dd}, J=8.5,2.0 \mathrm{~Hz}, 1 \mathrm{H}), 7.35(\mathrm{~d}, J=2.0 \mathrm{~Hz}, 1 \mathrm{H}), 7.31-7.27(\mathrm{~m}, 3 \mathrm{H}), 7.23-7.16$ $(\mathrm{m}, 5 \mathrm{H}), 7.14(\mathrm{~d}, J=2.7 \mathrm{~Hz}, 1 \mathrm{H}), 7.09-7.01(\mathrm{~m}, 5 \mathrm{H}), 6.97(\mathrm{~d}, J=8.3 \mathrm{~Hz}, 1 \mathrm{H}), 6.94(\mathrm{dd}$, $J=8.4,2.7 \mathrm{~Hz}, 1 \mathrm{H}), 5.05(\mathrm{~s}, 2 \mathrm{H}), 4.46(\mathrm{br} \mathrm{s}, 2 \mathrm{H}), 3.90(\mathrm{~s}, 3 \mathrm{H}), 3.85(\mathrm{~s}, 3 \mathrm{H}), 2.16(\mathrm{t}, J=$ $6.4 \mathrm{~Hz}, 1 \mathrm{H}) \mathrm{ppm} ;{ }^{13} \mathrm{C}$ NMR $\left(100 \mathrm{MHz}, \mathrm{CDCl}_{3}\right): \delta=190.7(\mathrm{CH}), 163.4(\mathrm{C}), 159.3(\mathrm{C})$, $156.1(\mathrm{C}), 151.3(\mathrm{C}), 143.1$ (C), $140.8(\mathrm{C}), 138.0(\mathrm{C}), 136.4(\mathrm{C}), 131.9(3 \times \mathrm{CH}), 131.3$ $(\mathrm{CH}), 131.1(\mathrm{C}), 131.0(\mathrm{C}), 130.1(\mathrm{C}), 129.4(\mathrm{C}), 128.5(2 \times \mathrm{CH}), 127.9(\mathrm{CH}), 127.6$ $(\mathrm{CH}), 127.4(\mathrm{CH}), 127.1(2 \times \mathrm{CH}), 125.0(\mathrm{CH}), 120.0(\mathrm{CH}), 119.9(\mathrm{CH}), 116.2(2 \times$ $\mathrm{CH}), 113.4(\mathrm{CH}), 113.3(\mathrm{CH}), 113.1(\mathrm{CH}), 111.6(\mathrm{CH}), 71.3\left(\mathrm{CH}_{2}\right), 63.7\left(\mathrm{CH}_{3}\right), 56.0$ $\left(\mathrm{CH}_{2}\right), 55.3\left(\mathrm{CH}_{3}\right)$ ppm; IR (neat): $v 3447 \mathrm{br}, 2925,2852,1690,1598,1573,1503,1460$ $1423,1271,1227,1155,1123,1024 ; \mathrm{MS}\left(\mathrm{ESI}^{+}\right) \mathrm{m} / z(\%): 595\left([\mathrm{M}+\mathrm{Na}]^{+}, 100 \%\right)$; HRMS $\left(\mathrm{ESI}^{+}\right): \mathrm{m} / z$ calcd for $\mathrm{C}_{37} \mathrm{H}_{32} \mathrm{NaO}_{6}[\mathrm{M}+\mathrm{Na}]^{+}$595.2091; found: 595.2077 . 
(E)-(4'-(3-(4-(1,3-Dithian-2-yl)phenoxy)-4-methoxystyryl)-2'-(benzyloxy)-4-methoxy[1,1'-biphenyl]-2-yl)methanol (25): A solution of arene $\mathbf{2 4}(1.31 \mathrm{~g}, 2.28 \mathrm{mmol})$, propan1,3-dithiol $(0.69 \mathrm{~mL}, 6.84 \mathrm{mmol})$ and PPTS $(130 \mathrm{mg}, 0.54 \mathrm{mmol})$ in DCM $(35 \mathrm{~mL})$ were heated at reflux for $18 \mathrm{~h}$ then cooled to RT and concentrated in vacuo. Purification by column chromatography $\left(60 \% \mathrm{Et}_{2} \mathrm{O} /\right.$ petrol $)$ afforded the title compound $\mathbf{2 5}$ as a white solid (1.39 g, 91\%); MP $72-73{ }^{\circ} \mathrm{C}\left(\mathrm{Et}_{2} \mathrm{O} /\right.$ petrol); ${ }^{1} \mathrm{H}$ NMR $\left(400 \mathrm{MHz}, \mathrm{CDCl}_{3}\right): \delta=7.43$ $(\mathrm{d}, J=8.7 \mathrm{~Hz}, 2 \mathrm{H}), 7.34-7.25(\mathrm{~m}, 4 \mathrm{H}), 7.23(\mathrm{~d}, J=2.1 \mathrm{~Hz}, 1 \mathrm{H}), 7.20-7.13(\mathrm{~m}, 6 \mathrm{H})$ $7.11(\mathrm{~d}, J=2.6 \mathrm{~Hz}, 1 \mathrm{H}), 7.06-6.97(\mathrm{~m}, 3 \mathrm{H}), 6.97-6.93(\mathrm{~m}, 2 \mathrm{H}), 6.91(\mathrm{dd}, J=8.4,2.7$ $\mathrm{Hz}, 1 \mathrm{H}), 5.18(\mathrm{~s}, 1 \mathrm{H}), 5.03($ br s, $2 \mathrm{H}), 4.43(\mathrm{br} \mathrm{s}, 2 \mathrm{H}), 3.88(\mathrm{~s}, 3 \mathrm{H}), 3.86(\mathrm{~s}, 3 \mathrm{H}), 3.12$ $3.03(\mathrm{~m}, 2 \mathrm{H}), 2.92($ app. td, $J=14.0,3.8 \mathrm{~Hz}, 2 \mathrm{H}), 2.19(\mathrm{~m}, 1 \mathrm{H}), 2.09(\mathrm{t}, J=6.1 \mathrm{~Hz}$, $1 \mathrm{H}), 1.93(\mathrm{~m}, 1 \mathrm{H}) \mathrm{ppm} ;{ }^{13} \mathrm{C} \mathrm{NMR}\left(100 \mathrm{MHz}, \mathrm{CDCl}_{3}\right): \delta=159.3(\mathrm{C}), 158.0(\mathrm{C}), 156.0$ (C), $151.4(\mathrm{C}), 144.6$ (C), $140.8(\mathrm{C}), 138.2(\mathrm{C}), 136.5$ (C), 133.1 (C), $131.8(\mathrm{CH}), 131.3$ $(\mathrm{CH}), 130.8(\mathrm{C}), 129.9(\mathrm{C}), 129.5(\mathrm{C}), 129.1(2 \times \mathrm{CH}), 128.5(2 \times \mathrm{CH}), 128.0(\mathrm{CH})$ $127.9(\mathrm{CH}), 127.2(2 \times \mathrm{CH}), 127.1(\mathrm{CH}), 123.8(\mathrm{CH}), 119.9(\mathrm{CH}), 119.2(\mathrm{CH}), 116.9(2$ $\times \mathrm{CH}), 113.4(\mathrm{CH}), 113.3(\mathrm{CH}), 112.9(\mathrm{CH}), 111.6(\mathrm{CH}), 71.3\left(\mathrm{CH}_{2}\right), 63.8\left(\mathrm{CH}_{2}\right), 56$. $\left(\mathrm{CH}_{3}\right), 55.3\left(\mathrm{CH}_{3}\right), 50.8(\mathrm{CH}), 32.2\left(2 \times \mathrm{CH}_{2}\right), 25.0\left(\mathrm{CH}_{2}\right) \mathrm{ppm}$; IR (solid): v $3437 \mathrm{br}$ 2933, 1602, 1503, 1441, 1422, 1272, 1227, 1167, 1025; MS (ESI+) $m / z(\%): 685$ $\left([\mathrm{M}+\mathrm{Na}]^{+}, 100 \%\right)$; HRMS $\left(\mathrm{ESI}^{+}\right): \mathrm{m} / z$ calcd for $\mathrm{C}_{40} \mathrm{H}_{38} \mathrm{NaO}_{5} \mathrm{~S}_{2}[\mathrm{M}+\mathrm{Na}]^{+} 685.2053$; found: 685.2061 .

(4'-(3-(4-(1,3-Dithian-2-yl)phenoxy)-4-methoxyphenethyl)-2'-(benzyloxy)-4-methoxy[1,1'-biphenyl]-2-yl)methanol (26): A solution of stilbene 25 (100 $\mathrm{mg}, 0.15 \mathrm{mmol}$ ), tosylhydrazone $(290 \mathrm{mg}, 1.57 \mathrm{mmol})$ and NaOAc $(130 \mathrm{~g}, 1.57 \mathrm{mmol})$ in $1: 1 \mathrm{v} / \mathrm{v}$ THF and water $(2 \mathrm{~mL}$ each) was heated at reflux for $18 \mathrm{~h}$ then cooled to RT. Water $(5 \mathrm{~mL})$ and $\mathrm{HCl}(2 \mathrm{M}, 2 \mathrm{~mL})$ were added and the reaction mixture was extracted with $\mathrm{Et}_{2} \mathrm{O}(3 \times$ $10 \mathrm{~mL}$ ). The combined organic phases were dried over $\mathrm{MgSO}_{4}$, concentrated in vacuo and purified by column chromatography (30\% EtOAc/petrol) to afford the title compound 26 as a white solid $(62 \mathrm{mg}, 62 \%)$; MP $78-79{ }^{\circ} \mathrm{C}$ (EtOAc/petrol); ${ }^{1} \mathrm{H}$ NMR $\left(400 \mathrm{MHz}, \mathrm{CDCl}_{3}\right): \delta=7.40(\mathrm{~d}, J=8.6 \mathrm{~Hz}, 2 \mathrm{H}), 7.30-7.26(\mathrm{~m}, 3 \mathrm{H}), 7.18-7.06(\mathrm{~m}$ $5 \mathrm{H}), 6.99-6.81(\mathrm{~m}, 8 \mathrm{H}), 5.15(\mathrm{~s}, 1 \mathrm{H}), 4.95(\mathrm{br} \mathrm{s}, 2 \mathrm{H}), 4.40(\mathrm{br} \mathrm{s}, 2 \mathrm{H}), 3.89(\mathrm{~s}, 3 \mathrm{H}), 3.82$ (s, 3H), 3.07 (br t, $J=13.1 \mathrm{~Hz}, 2 \mathrm{H}), 2.97-2.84(\mathrm{~m}, 6 \mathrm{H}), 2.21(\mathrm{br} \mathrm{s}, 1 \mathrm{H}), 2.15(\mathrm{~m}, 1 \mathrm{H})$ $1.95(\mathrm{~m}, 1 \mathrm{H}) \mathrm{ppm} ;{ }^{13} \mathrm{C} \mathrm{NMR}\left(100 \mathrm{MHz}, \mathrm{CDCl}_{3}\right): \delta=159.2(\mathrm{C}), 158.2(\mathrm{C}), 155.7(\mathrm{C})$ 149.9 (C), 144.0 (C), 142.4 (C), 140.8 (C), $136.6(\mathrm{C}), 134.7$ (C), 132.8 (C), $131.4(\mathrm{CH})$ $131.4(\mathrm{CH}), 129.7(\mathrm{C}), 129.0(2 \times \mathrm{CH}), 128.5(2 \times \mathrm{CH}), 128.3(\mathrm{C}), 127.8(\mathrm{CH}), 127.2(2$ $\times \mathrm{CH}), 125.0(\mathrm{CH}), 121.9(\mathrm{CH}), 121.8(\mathrm{CH}), 116.7(2 \times \mathrm{CH}), 114.3(\mathrm{CH}), 113.4(\mathrm{CH})$ $113.3(\mathrm{CH}), 112.9(\mathrm{CH}), 71.3\left(\mathrm{CH}_{2}\right), 63.8\left(\mathrm{CH}_{2}\right), 56.1\left(\mathrm{CH}_{3}\right), 55.3\left(\mathrm{CH}_{3}\right), 50.8(\mathrm{CH})$ $37.9\left(\mathrm{CH}_{2}\right), 36.8\left(\mathrm{CH}_{2}\right), 32.2\left(2 \times \mathrm{CH}_{2}\right), 25.1\left(\mathrm{CH}_{2}\right)$ ppm; IR (solid): v $3437 \mathrm{br}, 2933$ $1602,1503,1422,1272,1227,1167,1126,1025 ; \mathrm{MS}\left(\mathrm{ESI}^{+}\right) \mathrm{m} / \mathrm{z}(\%): 687\left([\mathrm{M}+\mathrm{Na}]^{+}\right.$ $100 \%$ ); HRMS $\left(\mathrm{ESI}^{+}\right): \mathrm{m} / z$ calcd for $\mathrm{C}_{40} \mathrm{H}_{40} \mathrm{O}_{5} \mathrm{~S}_{2}[\mathrm{M}+\mathrm{Na}]^{+}$687.2209; found: 687.2201

2-(4-(5-(2-(2-(Benzyloxy)-2'-(chloromethyl)-4'-methoxy-[1,1'-biphenyl]-4-yl)ethyl)-2methoxyphenoxy)phenyl)-1,3-dithiane (27): To a solution of benzyl alcohol 26 (75 mg, $0.11 \mathrm{mmol})$ and pyridine $(0.013 \mathrm{~mL}, 0.16 \mathrm{mmol})$ in $\mathrm{DCM}(5 \mathrm{~mL})$ at $0{ }^{\circ} \mathrm{C}$ was added thionyl chloride $(0.012 \mathrm{~mL}, 0.16 \mathrm{mmol})$. The temperature was raised to RT and after 18 $\mathrm{h}$, water $(5 \mathrm{~mL})$ was added. The aqueous phase was separated and extracted with $\mathrm{Et}_{2} \mathrm{O}$ $(3 \times 10 \mathrm{~mL})$ then the combined organic phases were dried over $\mathrm{MgSO}_{4}$, concentrated in vacuo and purified by column chromatography $\left(30 \% \mathrm{Et}_{2} \mathrm{O} /\right.$ petrol) to afford the title compound $\mathbf{2 7}$ as a white solid $(51 \mathrm{mg}, 67 \%)$.

Alternatively, to a solution of benzyl alcohol $26(0.25 \mathrm{~g}, 0.37 \mathrm{mmol})$ in DCM (18 mL) was added DBU $(0.17 \mathrm{~g}, 1.12 \mathrm{mmol}), \mathrm{LiCl}(157 \mathrm{mg}, 0.37 \mathrm{mmol})$ and $\mathrm{MsCl}(0.12 \mathrm{~g}, 1.12$ $\mathrm{mmol}$ ). After $18 \mathrm{~h}$ the reaction mixture was concentrated in vacuo and purified by column chromatography $\left(30 \% \mathrm{Et}_{2} \mathrm{O} /\right.$ petrol) to afford the title compound $\mathbf{2 7}$ as a white solid $(0.16 \mathrm{~g}, 63 \%)$; MP 59-60 ${ }^{\circ} \mathrm{C}$ (EtOAc/petrol); ${ }^{1} \mathrm{H}$ NMR $\left(400 \mathrm{MHz}, \mathrm{CDCl}_{3}\right): \delta=$ $7.40(\mathrm{~d}, J=8.7 \mathrm{~Hz}, 2 \mathrm{H}), 7.31-7.24(\mathrm{~m}, 3 \mathrm{H}), 7.19-7.13(\mathrm{~m}, 4 \mathrm{H}), 7.11(\mathrm{~d}, J=2.7 \mathrm{~Hz}$ $1 \mathrm{H}), 6.96-6.82(\mathrm{~m}, 7 \mathrm{H}), 6.79(\mathrm{~d}, J=0.8 \mathrm{~Hz}, 1 \mathrm{H}), 5.15(\mathrm{~s}, 1 \mathrm{H}), 4.98(\mathrm{~s}, 2 \mathrm{H}), 4.44(\mathrm{br}$ $2 \mathrm{H}), 3.88(\mathrm{~s}, 3 \mathrm{H}), 3.81(\mathrm{~s}, 3 \mathrm{H}), 3.12-3.01(\mathrm{~m}, 2 \mathrm{H}), 2.95-2.83(\mathrm{~m}, 6 \mathrm{H}), 2.18(\mathrm{~m}, 1 \mathrm{H})$ $1.91(\mathrm{~m}, 1 \mathrm{H}) \mathrm{ppm} ;{ }^{13} \mathrm{C} \mathrm{NMR}\left(100 \mathrm{MHz}, \mathrm{CDCl}_{3}\right): \delta=159.0(\mathrm{C}), 158.2(\mathrm{C}), 155.6(\mathrm{C})$ 149.9 (C), 144.1 (C), 142.6 (C), 137.2 (C), 137.1 (C), 134.7 (C), 132.8 (C), $131.9(\mathrm{CH})$ $131.5(\mathrm{CH}), 130.5(\mathrm{C}), 129.0(2 \times \mathrm{CH}), 128.4(2 \times \mathrm{CH}), 127.5(\mathrm{CH}), 127.1(\mathrm{C}), 126.7($ $\times \mathrm{CH}), 125.0(\mathrm{CH}), 121.9(\mathrm{CH}), 121.2(\mathrm{CH}), 116.7(2 \times \mathrm{CH}), 114.4(\mathrm{CH}), 113.9(\mathrm{CH})$ $113.5(\mathrm{CH}), 112.8(\mathrm{CH}), 70.3\left(\mathrm{CH}_{2}\right), 56.1\left(\mathrm{CH}_{3}\right), 55.3\left(\mathrm{CH}_{3}\right), 50.8(\mathrm{CH}), 44.8\left(\mathrm{CH}_{2}\right)$ $37.9\left(\mathrm{CH}_{2}\right), 36.8\left(\mathrm{CH}_{2}\right), 32.2\left(2 \times \mathrm{CH}_{2}\right), 25.1\left(\mathrm{CH}_{2}\right) \mathrm{ppm}$; IR (solid): v 2932, 1605, 1577 1501, 1270, 1223, 1166, 908, 729; $\mathrm{MS}\left(\mathrm{ESI}^{+}\right) \mathrm{m} / z(\%): 707\left(\left[\mathrm{M}\left\{{ }^{37} \mathrm{Cl}\right\}+\mathrm{Na}\right]^{+}, 40 \%\right), 705$ $\left(\left[\mathrm{M}\left\{{ }^{35} \mathrm{Cl}\right\}+\mathrm{Na}\right]^{+}, 100 \%\right)$; HRMS $\left(\mathrm{ESI}^{+}\right)$: calcd for $\mathrm{C}_{40} \mathrm{H}_{39} \mathrm{ClNaO}_{4} \mathrm{~S}_{2}[\mathrm{M}+\mathrm{Na}]^{+} 705.1870$ found: 705.1857 .

\section{1,2,3,17,18,29-Hexahydro-21-Benzyloxy-13,26-dimethoxy-1,5-dithia-11-oxa-7,10-}

etheno-12,16-metheno-19,22-ethenobenzo[w]spiro[5,19]pentacosadodecaene (28): To a solution of dithiane $27(120 \mathrm{mg}, 0.18 \mathrm{mmol})$ in THF $(15 \mathrm{~mL})$ at $-78{ }^{\circ} \mathrm{C}$ was added $n$ $\operatorname{BuLi}(2.44 \mathrm{M}$ in hexanes, $0.1 \mathrm{~mL}, 0.24 \mathrm{mmol})$. The reaction mixture was slowly warmed to RT and after $18 \mathrm{~h}$ sat. $\mathrm{NH}_{4} \mathrm{Cl}(5 \mathrm{~mL})$ and water $(10 \mathrm{~mL})$ were added. The aqueous phase was separated and extracted with $\mathrm{Et}_{2} \mathrm{O}(3 \times 20 \mathrm{~mL})$ then the combined organic phases were dried over $\mathrm{MgSO}_{4}$, concentrated in vacuo and purified by column chromatography ( $30 \% \mathrm{Et}_{2} \mathrm{O} /$ petrol) to afford the title compound $\mathbf{2 8}$ as a white solid (34 mg, 33\%); MP 83-85 ${ }^{\circ} \mathrm{C}\left(\mathrm{Et}_{2} \mathrm{O} /\right.$ petrol); ${ }^{1} \mathrm{H}$ NMR $\left(400 \mathrm{MHz}, \mathrm{CDCl}_{3}\right): \delta=7.73$ (br s, $1 \mathrm{H}), 7.55(\mathrm{br} \mathrm{s}, 1 \mathrm{H}), 7.33-7.24(\mathrm{~m}, 4 \mathrm{H}), 7.21-7.17(\mathrm{~m}, 2 \mathrm{H}), 7.00(\mathrm{~d}, J=8.4 \mathrm{~Hz}, 1 \mathrm{H})$ $6.90(\mathrm{~d}, J=8.6 \mathrm{~Hz}, 1 \mathrm{H}), 6.88-6.78(\mathrm{~m}, 5 \mathrm{H}), 6.52(\mathrm{~d}, J=1.3 \mathrm{~Hz}, 1 \mathrm{H}), 6.34(\mathrm{dd}, J=7.6$ $1.6 \mathrm{~Hz}, 1 \mathrm{H}), 5.69(\mathrm{~d}, J=2.1 \mathrm{~Hz}, 1 \mathrm{H}), 4.87(\mathrm{br}, 2 \mathrm{H}), 3.95(\mathrm{~s}, 3 \mathrm{H}), 3.93(\mathrm{~s}, 3 \mathrm{H}), 3.48(\mathrm{~s}$ 2H), 2.90-2.77 (br, 2H), 2.76-2.63 (br, 2H), 2.61-2.45 (br, 4H), 2.19-1.88 (br, 2H) ppm; ${ }^{13} \mathrm{C}$ NMR $\left(100 \mathrm{MHz}, \mathrm{CDCl}_{3}\right): \delta=157.5(\mathrm{C}), 154.8(\mathrm{C}), 153.3(\mathrm{C}), 147.6(\mathrm{C})$ 147.1 (C), 141.2 (C), 139.4 (C), 137.1 (C), 133.8 (C), 133.7 (C), $133.4(\mathrm{C}), 132.1(\mathrm{CH})$ $131.7(\mathrm{CH}), 130.3(\mathrm{CH}), 129.5(\mathrm{CH}), 128.4(\mathrm{C}), 128.3(2 \times \mathrm{CH}), 127.5(\mathrm{CH}), 126.8(2 \times$ $\mathrm{CH}), 122.5(\mathrm{CH}), 122.1(\mathrm{CH}), 122.1(\mathrm{CH}), 121.6(\mathrm{CH}), 118.5(\mathrm{CH}), 116.7(\mathrm{CH}), 113.5$ $(\mathrm{CH}), 113.3(\mathrm{CH}), 112.0(\mathrm{CH}), 70.1\left(\mathrm{CH}_{2}\right), 58.6(\mathrm{C}), 56.2\left(\mathrm{CH}_{3}\right), 55.4\left(\mathrm{CH}_{3}\right), 49.8$ $\left(\mathrm{CH}_{2}\right), 37.6\left(\mathrm{CH}_{2}\right), 36.4\left(\mathrm{CH}_{2}\right), 28.7\left(\mathrm{CH}_{2}\right), 28.1\left(\mathrm{CH}_{2}\right), 25.1\left(\mathrm{CH}_{2}\right) \mathrm{ppm}$; IR (solid): v 2926, 1604, 1513, 1494, 1420, 1261, 1230, 1165, 1128, 733; MS $\left(\mathrm{ESI}^{+}\right) \mathrm{m} / \mathrm{z}(\%): 669$ $\left([\mathrm{M}+\mathrm{Na}]^{+}, 100 \%\right)$; HRMS $\left(\mathrm{ESI}^{+}\right)$: calcd for $\mathrm{C}_{40} \mathrm{H}_{38} \mathrm{NaO}_{4} \mathrm{~S}_{2}[\mathrm{M}+\mathrm{Na}]^{+}$669.2104; found: 669.2098 .
Riccardin C dimethyl ether (12): ${ }^{[19,21]}$ To a solution of $\mathrm{NiCl}_{2} \cdot 6 \mathrm{H}_{2} \mathrm{O}(170 \mathrm{mg}, 0.74 \mathrm{mmol})$ in DMF $(1 \mathrm{~mL})$ was added sequentially a solution of dithiane $28(30 \mathrm{mg}, 0.046 \mathrm{mmol})$ in THF $(1 \mathrm{~mL})$ and $\mathrm{NaBH}_{4}(55 \mathrm{mg}, 1.47 \mathrm{mmol})$. After $18 \mathrm{~h}$, the mixture was filtered through Celite $^{\odot}$ and water $(5 \mathrm{~mL})$ was added. The aqueous phase was separated and extracted with $\mathrm{Et}_{2} \mathrm{O}(3 \times 10 \mathrm{~mL})$, then the combined organic phases were dried over $\mathrm{MgSO}_{4}$ and concentrated in vacuo. The crude material was next dissolved in EtOH (3 $\mathrm{mL})$ and DCM $(3 \mathrm{~mL})$ and $10 \%$ Pd-C $(4.8 \mathrm{mg})$ was added. The flask was evacuated and purged with argon three times then evacuated and filled with hydrogen twice. After a further $18 \mathrm{~h}$, the reaction mixture was filtered through Celite ${ }^{\odot}$, concentrated in vacuo and purified by column chromatography (35\% $\mathrm{Et}_{2} \mathrm{O} / \mathrm{h}$ xane) to afford the title compound 12 as a white solid $(15 \mathrm{mg}, 71 \%)$; MP $140-141{ }^{\circ} \mathrm{C}\left(\mathrm{Et}_{2} \mathrm{O} / \mathrm{hexane}\right)$ [Lit oil $\left.{ }^{[19]}\right] ;{ }^{1} \mathrm{H}$ NMR $\left(400 \mathrm{MHz}, \mathrm{CDCl}_{3}\right): \delta=7.11(\mathrm{~d}, J=8.4 \mathrm{~Hz}, 1 \mathrm{H}), 7.03(\mathrm{~d}, J=2.6 \mathrm{~Hz}$ $1 \mathrm{H}), 6.92-6.76(\mathrm{~m}, 5 \mathrm{H}), 6.89(\mathrm{~d}, J=7.9 \mathrm{~Hz}, 1 \mathrm{H}), 6.82(\mathrm{~d}, J=7.6 \mathrm{~Hz}, 1 \mathrm{H}), 6.78$ (dd, $J=$ $8.4,1.5 \mathrm{~Hz}, 1 \mathrm{H}), 6.44(\mathrm{~d}, J=0.7 \mathrm{~Hz}, 1 \mathrm{H}), 6.30(\mathrm{~d}, J=7.6 \mathrm{~Hz}, 1 \mathrm{H}), 5.44(\mathrm{~d}, J=1.5 \mathrm{~Hz}$, $1 \mathrm{H}), 4.79(\mathrm{~s}, 1 \mathrm{H}), 3.95(\mathrm{~s}, 3 \mathrm{H}), 3.91(\mathrm{~s}, 3 \mathrm{H}), 3.15-3.02(\mathrm{~m}, 1 \mathrm{H}), 3.02-2.83(\mathrm{~m}, 2 \mathrm{H})$ $2.83-2.58(\mathrm{~m}, 5 \mathrm{H}) \mathrm{ppm} ;{ }^{13} \mathrm{C} \mathrm{NMR}\left(100 \mathrm{MHz}, \mathrm{CDCl}_{3}\right): \delta=159.9(\mathrm{C}), 153.0(\mathrm{C}), 151.9$ (C), 148.6 (C), 146.9 (C), 143.6 (C), 142.0 (C), 139.3 (C), 133.8 (C), $132.5(\mathrm{CH}), 131.5$ $(\mathrm{CH}), 129.3(\mathrm{CH}), 129.3(\mathrm{CH}), 128.0(\mathrm{C}), 124.5(\mathrm{C}), 122.7(\mathrm{CH}), 122.5(\mathrm{CH}), 121.6$ $(\mathrm{CH}), 121.4(\mathrm{CH}), 116.6(\mathrm{CH}), 116.3(\mathrm{CH}), 115.9(\mathrm{CH}), 112.6(\mathrm{CH}), 111.8(\mathrm{CH}), 56.1$ $\left(\mathrm{CH}_{3}\right), 55.3\left(\mathrm{CH}_{3}\right), 38.2\left(\mathrm{CH}_{2}\right), 37.7\left(\mathrm{CH}_{2}\right), 37.0\left(\mathrm{CH}_{2}\right), 35.2\left(\mathrm{CH}_{2}\right)$ ppm; IR (solid): v $3467 \mathrm{br}, 2927,2853,1605,1506,1420,1260,1229,1164,1127$; MS $\left(\mathrm{ESI}^{+}\right) \mathrm{m} / \mathrm{z}(\%)$ : $453\left([\mathrm{M}+\mathrm{H}]^{+}, 100 \%\right)$; HRMS $\left(\mathrm{ESI}^{+}\right)$: calcd for $\mathrm{C}_{30} \mathrm{H}_{29} \mathrm{O}_{4}[\mathrm{M}+\mathrm{H}]^{+} 453.2060$; found: 453.2060 .

Riccardin C (10): To a solution of riccardin C dimethyl ether $12(7.5 \mathrm{mg}, 0.016 \mathrm{mmol})$ in DCM $(2 \mathrm{~mL})$ was added boron tribromide $(1 \mathrm{M}$ solution in DCM, $0.16 \mathrm{~mL}, 0.16$ $\mathrm{mmol})$. After $5 \mathrm{~h}$, water $(5 \mathrm{~mL})$ was added then the aqueous phase was separated and extracted with $\mathrm{Et}_{2} \mathrm{O}(3 \times 10 \mathrm{~mL})$. The combined organic phases were dried over $\mathrm{MgSO}_{4}$, concentrated in vacuo and purified by column chromatography $\left(60 \% \mathrm{Et}_{2} \mathrm{O} /\right.$ petrol $)$ to afford the title compound $\mathbf{1 0}$ as a white solid $(4.9 \mathrm{mg}, 71 \%)$. All spectroscopic and physical data matched that previously reported by us and others. ${ }^{[3,4,8-14]}$

5-Formyl-2-hydroxyphenyl trifluoromethanesulfonate $(29)$ : To a cooled $\left(0{ }^{\circ} \mathrm{C}\right)$ solution of phenol 13 (10.0 g, $72.4 \mathrm{mmol})$ and pyridine $(7.03 \mathrm{~mL}, 86.88 \mathrm{mmol})$ in DCM (240 $\mathrm{mL}$ ) was added $\mathrm{Tf}_{2} \mathrm{O}(12.18 \mathrm{~mL}, 72.4 \mathrm{mmol})$ over $5 \mathrm{~min}$. The reaction was allowed to warm to $\mathrm{RT}$ and after $13 \mathrm{~h}$, sat. $\mathrm{NH}_{4} \mathrm{Cl}(100 \mathrm{~mL})$ and water $(100 \mathrm{~mL})$ were added. The aqueous phase was separated and extracted with DCM $(3 \times 100 \mathrm{~mL})$, then the combined organic phases were dried over $\mathrm{MgSO}_{4}$, concentrated in vacuo and purified by column chromatography (50\% $\mathrm{Et}_{2} \mathrm{O}$ /cyclohexane) to afford the title compound $\mathbf{2 9}$ as a colourless oil $(14.65 \mathrm{~g}, 74 \%)$; ${ }^{1} \mathrm{H}$ NMR $\left(400 \mathrm{MHz}, \mathrm{CDCl}_{3}\right): \delta=10.03(\mathrm{~s}, 1 \mathrm{H}), 8.04(\mathrm{dd}$ $J=8.4,2.0 \mathrm{~Hz}, 1 \mathrm{H}), 8.01(\mathrm{~d}, J=1.8 \mathrm{~Hz}, 1 \mathrm{H}), 7.72(\mathrm{~d}, J=8.4 \mathrm{~Hz}, 1 \mathrm{H}) ;{ }^{13} \mathrm{C}$ NMR $\left(100 \mathrm{MHz}, \mathrm{CDCl}_{3}\right): \delta=188.3(\mathrm{CH}), 144.2(\mathrm{C}), 141.2(\mathrm{C}), 136.9(\mathrm{C}), 130.7(\mathrm{CH}), 124.5$ $(\mathrm{CH}), 123.7(\mathrm{CH}), 118.5\left(\mathrm{q}, J=320.6 \mathrm{~Hz}, \mathrm{CF}_{3}\right) \mathrm{ppm} ;{ }^{19} \mathrm{~F}$ NMR $\left(376 \mathrm{MHz}, \mathrm{CDCl}_{3}\right): \delta=$ -73.83 ppm; IR (film): v 3528, 2979, 1605, 1417, 1205, 1134, 1096, 907, $864 \mathrm{~cm}^{-1}$; MS $\left(\mathrm{EI}^{+}\right) \mathrm{m} / \mathrm{z}(\%): 270\left(\mathrm{M}^{+}, 4 \%\right), 149(87 \%), 69(100 \%)$.

2-(Benzyloxy)-5-formylphenyl trifluoromethanesulfonate (30): To a solution of $\mathbf{2 9}$ (22.5 $\mathrm{g}, 83.28 \mathrm{mmol})$ in $\mathrm{MeCN}(200 \mathrm{~mL})$ were added potassium carbonate $(17.26 \mathrm{~g}, 124.92$ mmol) and benzyl bromide $(11.89 \mathrm{~mL}, 99.94 \mathrm{mmol})$. After $13 \mathrm{~h}$, water $(100 \mathrm{~mL})$ and $\mathrm{Et}_{2} \mathrm{O}(50 \mathrm{~mL})$ were added. The aqueous phase was separated and extracted with $\mathrm{Et}_{2} \mathrm{O}(2$ $\times 50 \mathrm{~mL}$ ) then the combined organic phases were dried over $\mathrm{MgSO}_{4}$, concentrated in vacuo and purified by column chromatography $\left(20-25 \% \mathrm{Et}_{2} \mathrm{O} /\right.$ petrol) to afford the title compound 30 as an orange oil $(17.1 \mathrm{~g}, 72 \%) ;{ }^{1} \mathrm{H}$ NMR $\left(400 \mathrm{MHz}, \mathrm{CDCl}_{3}\right): \delta=9.89(\mathrm{~s}$ $1 \mathrm{H}), 7.85(\mathrm{dd}, J=8.5,1.9 \mathrm{~Hz}, 1 \mathrm{H}), 7.79(\mathrm{~d}, J=1.9 \mathrm{~Hz}, 1 \mathrm{H}), 7.49-7.35(\mathrm{~m}, 5 \mathrm{H}), 7.22(\mathrm{~d}$ $J=8.5 \mathrm{~Hz}, 1 \mathrm{H}), 5.29(\mathrm{~s}, 2 \mathrm{H}) \mathrm{ppm} ;{ }^{13} \mathrm{C} \mathrm{NMR}\left(100 \mathrm{MHz}, \mathrm{CDCl}_{3}\right): \delta=189.0(\mathrm{CH}), 155.4$ (C), $139.3(\mathrm{C}), 134.5(\mathrm{C}), 131.8(\mathrm{CH}), 130.1(\mathrm{C}), 128.8(2 \times \mathrm{CH}), 128.6(\mathrm{C}), 127.3(2 \times$ $\mathrm{CH}), 123.0(\mathrm{CH}), 114.2(\mathrm{CH}), 118.6\left(\mathrm{q}, J=320.6 \mathrm{~Hz}, \mathrm{CF}_{3}\right), 71.6\left(\mathrm{CH}_{2}\right) \mathrm{ppm}$; IR (film): $v$ 1697, 1607,1508,1423,1318,1278, 1247, 1209, 1137, 1090, 905, $725 \mathrm{~cm}^{-1}$; MS $\left(\mathrm{ESI}^{+}\right) \mathrm{m} / \mathrm{z}(\%): 361\left(\mathrm{MH}^{+}, 100 \%\right) ; \mathrm{HRMS}\left(\mathrm{ESI}^{+}\right): \mathrm{m} / z$ calcd for $\mathrm{C}_{15} \mathrm{H}_{11} \mathrm{~F}_{3} \mathrm{NaO}_{5} \mathrm{~S}$ $[\mathrm{M}+\mathrm{Na}]^{+}$383.0171; found: 383.0180

2-(Benzyloxy)-5-ethenylphenyl trifluoromethanesulfonate (31): To a cooled $\left(0{ }^{\circ} \mathrm{C}\right)$ solution of aldehyde $\mathbf{3 0}(5.00 \mathrm{~g}, 13.88 \mathrm{mmol})$ in THF $(125 \mathrm{~mL})$ were added sequentially sodium hydride (1.66 g, $41.63 \mathrm{mmol})$ and methyltriphenylphosph-onium bromide (7.43 $\mathrm{g}, 20.82 \mathrm{mmol}$ ). The reaction mixture was warmed to RT and after $30 \mathrm{~min}$ was concentrated in vacuo and purified by column chromatography (5-10\% $\mathrm{Et}_{2} \mathrm{O} /$ petrol) to afford the title compound $\mathbf{3 1}$ as a yellow oil $(3.81 \mathrm{~g}, 76 \%)$. ${ }^{1} \mathrm{H} \mathrm{NMR}\left(400 \mathrm{MHz}, \mathrm{CDCl}_{3}\right)$ : $\delta=7.50(\mathrm{~d}, J=7.2 \mathrm{~Hz}, 2 \mathrm{H}), 7.44(\mathrm{t}, J=7.5 \mathrm{~Hz}, 2 \mathrm{H}), 7.38(\mathrm{t}, J=7.5 \mathrm{~Hz}, 1 \mathrm{H}), 7.35-7.30$ $(\mathrm{m}, 2 \mathrm{H}), 7.04(\mathrm{~d}, J=8.8 \mathrm{~Hz}, 1 \mathrm{H}), 6.65(\mathrm{dd}, J=17.5,10.9 \mathrm{~Hz}, 1 \mathrm{H}), 5.69(\mathrm{~d}, J=17.6 \mathrm{~Hz}$, $1 \mathrm{H}), 5.29(\mathrm{~d}, J=10.9 \mathrm{~Hz}, 1 \mathrm{H}), 5.20(\mathrm{~s}, 2 \mathrm{H}) \mathrm{ppm} ;{ }^{13} \mathrm{C} \mathrm{NMR}\left(100 \mathrm{MHz}, \mathrm{CDCl}_{3}\right): \delta=$ $149.9(\mathrm{C}), 139.0(\mathrm{C}), 135.6(\mathrm{C}), 134.6(\mathrm{CH}), 131.5(\mathrm{C}), 128.6(2 \times \mathrm{CH}), 128.2(\mathrm{CH})$ $127.2(2 \times \mathrm{CH}), 126.9(\mathrm{CH}), 119.8(\mathrm{CH}), 114.4(\mathrm{CH}), 114.0\left(\mathrm{CH}_{2}\right), 118.7(\mathrm{q}, J=320.6$ $\left.\mathrm{Hz}, \mathrm{CF}_{3}\right), 71.0\left(\mathrm{CH}_{2}\right) \mathrm{ppm}$; IR (film): v 2953, 1615, 1509, 1419, 1270, 1247, 1201, 1137 , 1092, 942, $734 \mathrm{~cm}^{-1} ; \mathrm{MS}\left(\mathrm{EI}^{+}\right) \mathrm{m} / \mathrm{z}(\%): 358\left(\mathrm{M}^{+}, 10 \%\right), 91(100 \%)$; HRMS $\left(\mathrm{ESI}^{+}\right): \mathrm{m} / \mathrm{z}$ calcd for $\mathrm{C}_{16} \mathrm{H}_{13} \mathrm{~F}_{3} \mathrm{NaO}_{4} \mathrm{~S}[\mathrm{M}+\mathrm{Na}]^{+}$381.0379; found: 381.0389 .

2'-(Benzyloxy)-4-methoxy-5'-vinyl-[1,1'-biphenyl]-2-carbaldehyde (32): To a solution of triflate $31(2.49 \mathrm{~g}, 6.95 \mathrm{mmol})$ and boronic acid $16(1.5 \mathrm{~g}, 8.38 \mathrm{mmol})$ in a mixed solvent system of $\mathrm{PhMe} / \mathrm{EtOH} /$ water $(150 / 75 / 75 \mathrm{~mL})$ was added $\mathrm{Cs}_{2} \mathrm{CO}_{3}(4.53 \mathrm{~g}, 13.9$ mmol). The resulting mixture was degassed with argon for $20 \mathrm{~min}$ then $\mathrm{Pd}\left(\mathrm{PPh}_{3}\right)_{4}(0.52$ $\mathrm{g}, 0.45 \mathrm{mmol}$ ) was added and the solution was heated at reflux for $14 \mathrm{~h}$. On cooling to $\mathrm{RT}$, water $(100 \mathrm{~mL})$ was added and the aqueous phase was separated and extracted with $\mathrm{Et}_{2} \mathrm{O}(3 \times 100 \mathrm{~mL})$. The combined organic phases were dried over $\mathrm{MgSO}_{4}$, concentrated in vacuo and purified by column chromatography (10-15\% $\mathrm{Et}_{2} \mathrm{O} /$ cyclohexane) to afford the title compound 32 as a yellow oil $(2.11 \mathrm{~g}, 90 \%) ;{ }^{1} \mathrm{H} \mathrm{NMR}\left(400 \mathrm{MHz}, \mathrm{CDCl}_{3}\right): \delta=$ $9.81(\mathrm{~s}, 1 \mathrm{H}), 7.48(\mathrm{~d}, J=2.8 \mathrm{~Hz}, 1 \mathrm{H}), 7.36(\mathrm{dd}, J=8.5,2.3 \mathrm{~Hz}, 1 \mathrm{H}), 7.31-7.21(\mathrm{~m}, 5 \mathrm{H})$ $7.18(\mathrm{dd}, J=8.5,2.8 \mathrm{~Hz}, 1 \mathrm{H}), 7.16-7.10(\mathrm{~m}, 2 \mathrm{H}), 6.94(\mathrm{~d}, J=8.4 \mathrm{~Hz}, 1 \mathrm{H}), 6.66(\mathrm{dd}, J=$ 
17.6, $10.9 \mathrm{~Hz}, 1 \mathrm{H}), 5.62(\mathrm{dd}, J=17.6,0.6 \mathrm{~Hz}, 1 \mathrm{H}), 5.16(\mathrm{dd}, J=10.9,0.6 \mathrm{~Hz}, 1 \mathrm{H}), 5.00$ (br, $2 \mathrm{H}), 3.87(\mathrm{~s}, 3 \mathrm{H}) ;{ }^{13} \mathrm{C}$ NMR $\left(100 \mathrm{MHz}, \mathrm{CDCl}_{3}\right): \delta=192.4(\mathrm{CH}), 159.2(\mathrm{C}), 155.6$ (C), $136.5(\mathrm{C}), 135.7(\mathrm{CH}), 134.9(\mathrm{C}), 134.7(\mathrm{C}), 132.5(\mathrm{CH}), 130.9(\mathrm{C}), 129.6(\mathrm{CH})$ $128.4(2 \times \mathrm{CH}), 127.8(\mathrm{CH}), 127.4(\mathrm{CH}), 127.4(\mathrm{C}), 126.9(2 \times \mathrm{CH}), 121.2(\mathrm{CH}), 112.8$ $(\mathrm{CH}), 112.7\left(\mathrm{CH}_{2}\right), 109.4(\mathrm{CH}), 70.5\left(\mathrm{CH}_{2}\right), 55.6\left(\mathrm{CH}_{3}\right)$ ppm; IR (film): v 2927, 2854 $1698,1603,1489,1455,1419,1393,1315,1269,1225,1161,1148,1043,1016 \mathrm{~cm}^{-1}$ MS $\left(\mathrm{ESI}^{+}\right) \mathrm{m} / \mathrm{z}(\%): 344\left([\mathrm{M}+\mathrm{Na}]^{+}, 100 \%\right)$.

(2'-(Benzyloxy)-4-methoxy-5'-vinyl-[1,1'-biphenyl]-2-yl)methanol (35): To a suspension of aldehyde $32(11.85 \mathrm{~g}, 34.4 \mathrm{mmol})$ in methanol $(140 \mathrm{~mL})$ and DCM $(140 \mathrm{~mL})$ at $0{ }^{\circ} \mathrm{C}$ was added $\mathrm{NaBH}_{4}(2.60 \mathrm{~g}, 68.8 \mathrm{mmol})$. The reaction mixture warmed to RT and after 2 $\mathrm{h}$, water $(100 \mathrm{~mL})$ was added. The aqueous phase was separated and extracted with $\mathrm{Et}_{2} \mathrm{O}(3 \times 250 \mathrm{~mL})$ then the combined organic phases were dried over $\mathrm{MgSO}_{4}$ concentrated in vacuo and purified by column chromatography (30-40\% $\mathrm{Et}_{2} \mathrm{O} /$ petrol) to afford the title compound $\mathbf{3 5}$ as a colourless oil $(11.90 \mathrm{~g}, 34.4 \mathrm{mmol}, 99 \%) ;{ }^{1} \mathrm{H}$ NMR $\left(400 \mathrm{MHz}, \mathrm{CDCl}_{3}\right): \delta=7.38(\mathrm{dd}, J=8.5,2.3 \mathrm{~Hz}, 1 \mathrm{H}), 7.34-7.27(\mathrm{~m}, 4 \mathrm{H}), 7.22-7.18$ $(\mathrm{m}, 3 \mathrm{H}), 7.16(\mathrm{~d}, J=2.7 \mathrm{~Hz}, 1 \mathrm{H}), 7.02(\mathrm{~d}, J=8.6 \mathrm{~Hz}, 1 \mathrm{H}), 6.95(\mathrm{dd}, J=8.3,2.7 \mathrm{~Hz}$ $1 \mathrm{H}), 6.71(\mathrm{dd}, J=17.6,10.9, \mathrm{~Hz}, 1 \mathrm{H}), 5.68(\mathrm{~d}, J=17.6 \mathrm{~Hz}, 1 \mathrm{H}), 5.21(\mathrm{~d}, J=11.0 \mathrm{~Hz}$ 1H), 5.03 (br, 2H), 4.56-4.36 (br, 2H), 3.90 (s, 3H), 2.24 (br. s, $1 \mathrm{H}) \mathrm{ppm} ;{ }^{13} \mathrm{C}$ NMR $\left(100 \mathrm{MHz}, \mathrm{CDCl}_{3}\right): \delta=159.3(\mathrm{C}), 155.5(\mathrm{C}), 140.7(\mathrm{C}), 136.4(\mathrm{C}), 135.9(\mathrm{CH}), 131.2$ $(\mathrm{CH}), 131.2(\mathrm{C}), 130.6(\mathrm{C}), 129.4(\mathrm{C}), 129.3(\mathrm{CH}), 128.5(2 \times \mathrm{CH}), 127.9(\mathrm{CH}), 127.0$ $(2 \times \mathrm{CH}), 126.6(\mathrm{CH}), 113.7(\mathrm{CH}), 113.3(\mathrm{CH}), 113.2(\mathrm{CH}), 112.4\left(\mathrm{CH}_{2}\right), 71.1\left(\mathrm{CH}_{2}\right)$ $63.6\left(\mathrm{CH}_{2}\right), 55.3\left(\mathrm{CH}_{3}\right) \mathrm{ppm}$; IR (film): v $3450 \mathrm{br}, 2939,1604,1489,1266,1228,1012$ 904, $724 \mathrm{~cm}^{-1}$; MS $\left(\mathrm{ESI}^{+}\right) \mathrm{m} / \mathrm{z}(\%): 369\left([\mathrm{M}+\mathrm{Na}\}^{+}, 100 \%\right) ; \mathrm{HRMS}\left(\mathrm{ESI}^{+}\right): \mathrm{m} / z$ calcd fo $\mathrm{C}_{23} \mathrm{H}_{22} \mathrm{NaO}_{3}[\mathrm{M}+\mathrm{Na}]^{+}$369.1461; found: 369.1469 .

(E)-4-(5-(2-(6-(Benzyloxy)-2'-(hydroxymethyl)-4'-methoxy-[1,1'-biphenyl]-3-yl)vinyl)-2methoxyphenoxy)benzaldehyde (34): A solution of styrene $35(4.78 \mathrm{~g}, 13.4 \mathrm{mmol})$, iodide $23(5.14 \mathrm{~g}, 14.8 \mathrm{mmol})$ and potassium phosphate $(4.01 \mathrm{~g}, 18.8 \mathrm{mmol})$ in DMA $(55 \mathrm{~mL})$ was degassed by sonication for 20 min under argon then $\mathrm{Pd}(\mathrm{OAc})_{2}(150 \mathrm{mg}$, $0.67 \mathrm{mmol}$ ) was added. The reaction mixture was heated at reflux for $18 \mathrm{~h}$ then cooled to RT and partitioned between water $(100 \mathrm{~mL})$ was and EtOAc $(50 \mathrm{~mL})$. The aqueous phase was separated and extracted with EtOAc $(2 \times 50 \mathrm{~mL})$ then the combined organic phases were dried over $\mathrm{MgSO}_{4}$, concentrated in vacuo and purified by column chromatography $\left(65 \% \mathrm{Et}_{2} \mathrm{O} /\right.$ petrol) to afford the title compound $\mathbf{3 4}$ as a white solid $(5.84 \mathrm{~g}, 75 \%)$; MP $70-71{ }^{\circ} \mathrm{C}\left(\mathrm{Et}_{2} \mathrm{O} /\right.$ petrol) ${ }^{1} \mathrm{H}$ NMR $\left(400 \mathrm{MHz}, \mathrm{CDCl}_{3}\right): \delta=9.91(\mathrm{~s}$ $1 \mathrm{H}), 7.84(\mathrm{~d}, J=8.8 \mathrm{~Hz}, 2 \mathrm{H}), 7.40(\mathrm{dd}, J=8.6,2.3 \mathrm{~Hz}, 1 \mathrm{H}), 7.34(\mathrm{~d}, J=2.3 \mathrm{~Hz}, 1 \mathrm{H})$ $7.33-7.26(\mathrm{~m}, 5 \mathrm{H}), 7.20-7.16(\mathrm{~m}, 3 \mathrm{H}), 7.12(\mathrm{~d}, J=2.7 \mathrm{~Hz}, 1 \mathrm{H}), 7.04-6.98(\mathrm{~m}, 4 \mathrm{H})$ 6.94-6.90 (m, 3H), $5.02(\mathrm{br}, 2 \mathrm{H}), 4.35-4.51(\mathrm{br}, 2 \mathrm{H}), 3.88(\mathrm{~s}, 3 \mathrm{H}), 3.81(\mathrm{~s}, 3 \mathrm{H}), 2.15(\mathrm{~s}$ $1 \mathrm{H}) \mathrm{ppm} ;{ }^{13} \mathrm{C}$ NMR $\left(100 \mathrm{MHz}, \mathrm{CDCl}_{3}\right): \delta=190.8(\mathrm{CH}), 163.4(\mathrm{C}), 159.4(\mathrm{C}), 155.4$ (C), $151.0(\mathrm{C}), 143.1(\mathrm{C}), 140.7$ (C), $136.4(\mathrm{C}), 131.9(2 \times \mathrm{CH}), 131.4(\mathrm{C}), 131.3(\mathrm{CH})$ $130.9(\mathrm{C}), 130.9(\mathrm{C}), 130.7(\mathrm{C}), 129.4(\mathrm{C}), 129.4(\mathrm{CH}), 128.5(2 \times \mathrm{CH}), 127.9(\mathrm{CH})$ $127.1(2 \times \mathrm{CH}), 127.0(\mathrm{C}), 126.9(\mathrm{CH}), 126.0(\mathrm{CH}), 124.7(\mathrm{CH}), 119.7(\mathrm{CH}), 116.2(2 \times$ $\mathrm{CH}), 113.9(\mathrm{CH}), 113.4(\mathrm{CH}), 113.3(\mathrm{CH}), 113.1(\mathrm{CH}), 71.2\left(\mathrm{CH}_{2}\right), 63.7\left(\mathrm{CH}_{2}\right), 56.0$ $\left(\mathrm{CH}_{3}\right), 55.3\left(\mathrm{CH}_{3}\right)$ ppm; IR (solid): $v 3438$ br, 2933, 2836, 1689, 1596, 1581, 1501, 1454, 1269, 1225, 1153, 725; MS $\left(\mathrm{ESI}^{+}\right) \mathrm{m} / \mathrm{z}(\%): 595\left([\mathrm{M}+\mathrm{Na}]^{+}, 100 \%\right)$; HRMS $\left(\mathrm{ESI}^{+}\right): \mathrm{m} / z$ calcd for $\mathrm{C}_{37} \mathrm{H}_{32} \mathrm{NaO}_{6}[\mathrm{M}+\mathrm{Na}]^{+} 595.2091$; found: 595.2082 .

(E)-(5'-(3-(4-(1,3-Dithian-2-yl)phenoxy)-4-methoxystyryl)-2'-(benzyloxy)-4-methoxy[1,1'-biphenyl]-2-yl)methanol (33): A solution of arene 34 (4.53 g, $7.93 \mathrm{~mol})$, propan1,3-dithiol (2.58 g, $23.8 \mathrm{mmol})$ and PPTS $(490 \mathrm{mg}, 1.90 \mathrm{mmol})$ in DCM $(88 \mathrm{~mL})$ was heated at reflux for $18 \mathrm{~h}$ then cooled to RT. Water $(30 \mathrm{~mL})$ and saturated aqueous $\mathrm{NaHCO}_{3}(100 \mathrm{~mL})$ were then added and the aqueous phase was extracted with $\mathrm{Et}_{2} \mathrm{O}(60$ $\mathrm{mL}$ ). The combined organic phases were dried over $\mathrm{MgSO}_{4}$, concentrated in vacuo and purified by column chromatography $\left(70 \% \mathrm{Et}_{2} \mathrm{O} /\right.$ petrol) to afford the title compound 33 as a white foamy solid $(4.79 \mathrm{~g}, 91 \%)$; $\mathrm{MP} 85-87{ }^{\circ} \mathrm{C}\left(\mathrm{Et}_{2} \mathrm{O} /\right.$ petrol $) ;{ }^{1} \mathrm{H} \mathrm{NMR}(400 \mathrm{MHz}$, $\left.\mathrm{CDCl}_{3}\right): \delta=7.43-7.36(\mathrm{~m}, 3 \mathrm{H}), 7.33(\mathrm{~d}, J=2.2 \mathrm{~Hz}, 1 \mathrm{H}), 7.30-7.25(\mathrm{~m}, 3 \mathrm{H}), 7.24(\mathrm{dd}$, $=8.5,2.2 \mathrm{~Hz}, 1 \mathrm{H}), 7.19-7.15(\mathrm{~m}, 4 \mathrm{H}), 7.12(\mathrm{~d}, J=2.7 \mathrm{~Hz}, 1 \mathrm{H}), 6.99(\mathrm{t}, J=8.1 \mathrm{~Hz}, 2 \mathrm{H})$, 6.94-6.87 (m, 5H), $5.16(\mathrm{~s}, 1 \mathrm{H}), 5.01(\mathrm{br}, 2 \mathrm{H}), 4.55-4.35(\mathrm{br}, 2 \mathrm{H}), 3.88(\mathrm{~s}, 3 \mathrm{H}), 3.84(\mathrm{~s}$ $3 \mathrm{H}), 3.11-3.01(\mathrm{~m}, 2 \mathrm{H}), 2.91(\mathrm{dt}, J=14.0,3.7 \mathrm{~Hz}, 2 \mathrm{H}), 2.21-2.11(\mathrm{~m}, 2 \mathrm{H}), 1.97-1.86$ (m, 1H) ppm; $\left.{ }^{13} \mathrm{C} \mathrm{NMR} \mathrm{(100} \mathrm{MHz,} \mathrm{CDCl}_{3}\right): \delta=159.3$ (C), 158.0 (C), 155.3 (C), 151.0 (C), $144.5(\mathrm{C}), 140.7(\mathrm{C}), 136.4(\mathrm{C}), 133.0(\mathrm{C}), 131.3(\mathrm{CH}), 131.1(\mathrm{C}), 130.9(\mathrm{C}), 130.8$ (C), $129.5(\mathrm{C}), 129.3(\mathrm{CH}), 129.0(2 \times \mathrm{CH}), 128.5(2 \times \mathrm{CH}), 127.1(\mathrm{CH}), 126.9(2 \times$ $\mathrm{CH}), 126.8(\mathrm{CH}), 126.6(\mathrm{CH}), 126.2(\mathrm{CH}), 123.5(\mathrm{CH}), 118.9(\mathrm{CH}), 116.9(2 \times \mathrm{CH})$, $113.9(\mathrm{CH}), 113.4(\mathrm{CH}), 113.2(\mathrm{CH}), 112.9(\mathrm{CH}), 71.2\left(\mathrm{CH}_{2}\right), 63.7\left(\mathrm{CH}_{2}\right), 56.0\left(\mathrm{CH}_{3}\right)$ $55.3\left(\mathrm{CH}_{3}\right), 50.7(\mathrm{CH}), 32.1\left(2 \times \mathrm{CH}_{2}\right), 25.0\left(\mathrm{CH}_{2}\right)$ ppm; IR (solid): v $3445 \mathrm{br}, 2933$ $2897,1605,1501,1454,1441,1422,1268,1222,1166,1124,1023,907,727$; MS (ESI+) $\mathrm{m} / \mathrm{z}(\%): 685\left([\mathrm{M}+\mathrm{Na}]^{+}, 100 \%\right)$; HRMS $\left(\mathrm{ESI}^{+}\right): \mathrm{m} / z$ calcd for $\mathrm{C}_{40} \mathrm{H}_{38} \mathrm{NaO}_{5} \mathrm{~S}_{2}$ $[\mathrm{M}+\mathrm{Na}]^{+}$685.2053; found: 685.2058

(5'-(3-(4-(1,3-Dithian-2-yl)phenoxy)-4-methoxyphenethyl)-2'-(benzyloxy)-4-methoxy-

[1,1'-biphenyl]-2-yl)methanol (36): A solution of stilbene $33(1.50 \mathrm{~g}, 2.26 \mathrm{mmol})$, tosylhydrazone $(4.21 \mathrm{~g}, 22.62 \mathrm{mmol})$ and $\mathrm{NaOAc}(1.86 \mathrm{~g}, 22.62 \mathrm{mmol})$ in $1: 1 \mathrm{v} / \mathrm{v}$ THF and water $(30 \mathrm{~mL}$ each) was heated at reflux for $18 \mathrm{~h}$ then cooled to RT. Saturated $\mathrm{K}_{2} \mathrm{CO}_{3}(50 \mathrm{~mL})$ and water $(10 \mathrm{~mL})$ were added and the reaction mixture was extracted with $\mathrm{Et}_{2} \mathrm{O}(3 \times 100 \mathrm{~mL})$. The combined organic phases were dried over $\mathrm{MgSO}_{4}$ concentrated in vacuo and purified by column chromatography $\left(50 \% \mathrm{Et}_{2} \mathrm{O} / \mathrm{petrol}\right)$ to afford the title compound 36 as a white solid $(1.37 \mathrm{~g}, 91 \%)$; MP $90-92{ }^{\circ} \mathrm{C}\left(\mathrm{Et}_{2} \mathrm{O} /\right.$ petrol); ${ }^{1} \mathrm{H}$ NMR $\left(400 \mathrm{MHz}, \mathrm{CDCl}_{3}\right): \delta=7.37(\mathrm{~d}, J=8.7 \mathrm{~Hz}, 2 \mathrm{H}), 7.31-7.27(\mathrm{~m}, 3 \mathrm{H}), 7.20$ $7.15(\mathrm{~m}, 2 \mathrm{H}), 7.13-7.10(\mathrm{~m}, 2 \mathrm{H}), 7.08(\mathrm{dd}, J=8.3,2.2 \mathrm{~Hz}, 1 \mathrm{H}), 6.95(\mathrm{~d}, J=8.4 \mathrm{~Hz}$ $1 \mathrm{H}), 6.93-6.89(\mathrm{~m}, 4 \mathrm{H}), 6.85(\mathrm{~d}, J=8.8 \mathrm{~Hz}, 2 \mathrm{H}), 6.81(\mathrm{br} \mathrm{s}, 1 \mathrm{H}), 5.14(\mathrm{~s}, 1 \mathrm{H}), 4.98(\mathrm{br}$ $2 \mathrm{H}), 4.48-4.29(\mathrm{br}, 2 \mathrm{H}), 3.89(\mathrm{~s}, 3 \mathrm{H}), 3.81(\mathrm{~s}, 3 \mathrm{H}), 3.14-2.98(\mathrm{~m}, 2 \mathrm{H}), 2.90(\mathrm{dt}, J=$ $14.1,3.7 \mathrm{~Hz}, 2 \mathrm{H}), 2.86(\mathrm{br} \mathrm{s}, 4 \mathrm{H}), 2.31(\mathrm{t}, J=6.2 \mathrm{~Hz}, 1 \mathrm{H}), 2.17(\mathrm{~m}, 1 \mathrm{H}), 1.92(\mathrm{~m}, 1 \mathrm{H})$ ppm; ${ }^{13} \mathrm{C}$ NMR $\left(100 \mathrm{MHz}, \mathrm{CDCl}_{3}\right): \delta=159.2(\mathrm{C}), 158.1(\mathrm{C}), 154.0(\mathrm{C}), 149.7(\mathrm{C})$ 143.9 (C), 140.7 (C), 136.7 (C), 134.6 (C), 134.5 (C), 132.7 (C), $131.8(\mathrm{CH}), 131.2$ $(\mathrm{CH}), 130.4(\mathrm{C}), 129.7(\mathrm{C}), 128.9(2 \times \mathrm{CH}), 128.5(\mathrm{CH}), 128.4(2 \times \mathrm{CH}), 127.8(\mathrm{CH})$ $127.1(2 \times \mathrm{CH}), 125.0(\mathrm{CH}), 121.8(\mathrm{CH}), 116.6(2 \times \mathrm{CH}), 113.9(\mathrm{CH}), 113.3(\mathrm{CH})$ $113.1(\mathrm{CH}), 112.7(\mathrm{CH}), 71.3\left(\mathrm{CH}_{2}\right), 63.6\left(\mathrm{CH}_{2}\right) 56.0\left(\mathrm{CH}_{3}\right), 55.3\left(\mathrm{CH}_{3}\right), 50.7(\mathrm{CH})$,
$36.9\left(\mathrm{CH}_{2}\right), 36.9\left(\mathrm{CH}_{2}\right), 32.1\left(2 \times \mathrm{CH}_{2}\right), 24.8\left(\mathrm{CH}_{2}\right)$ ppm; IR (solid): v $3447 \mathrm{br}, 2933$,

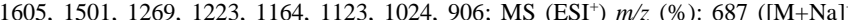
$100 \%$ ); HRMS $\left(\mathrm{ESI}^{+}\right): \mathrm{m} / z$ calcd for $\mathrm{C}_{40} \mathrm{H}_{40} \mathrm{O}_{5} \mathrm{~S}_{2}[\mathrm{M}+\mathrm{Na}]^{+}$687.2209; found: 687.2203 .

2-(4-(5-(2-(6-(Benzyloxy)-2'-(chloromethyl)-4'-methoxy-[1,1'-biphenyl]-3-yl)ethyl)-2methoxyphenoxy)phenyl)-1,3-dithiane (37): To a solution of benzyl alcohol $36(0.76 \mathrm{~g}$, $1.14 \mathrm{mmol})$ in DCM $(55 \mathrm{~mL})$ was added DBU $(0.52 \mathrm{~g}, 3.42 \mathrm{mmol}), \mathrm{LiCl}(0.053 \mathrm{~g}, 1.25$ $\mathrm{mmol})$ and $\mathrm{MsCl}(0.39 \mathrm{~g}, 3.42 \mathrm{mmol})$. After $18 \mathrm{~h}$ the reaction mixture was concentrated in vacuo and purified by column chromatography ( $30 \% \mathrm{Et}_{2} \mathrm{O} /$ petrol) to afford the title compound 37 as a colourless oil $(0.69 \mathrm{~g}, 88 \%) ;{ }^{1} \mathrm{H} \mathrm{NMR}\left(400 \mathrm{MHz}, \mathrm{CDCl}_{3}\right): \delta=7.35$ $(\mathrm{d}, J=8.7 \mathrm{~Hz}, 2 \mathrm{H}), 7.31-7.23(\mathrm{~m}, 3 \mathrm{H}), 7.20-7.17(\mathrm{~m}, 2 \mathrm{H}), 7.15(\mathrm{~d}, J=8.4 \mathrm{~Hz}, 1 \mathrm{H})$ $7.10(\mathrm{~d}, J=2.6 \mathrm{~Hz}, 1 \mathrm{H}), 7.05(\mathrm{dd}, J=8.3,2.3 \mathrm{~Hz}, 1 \mathrm{H}), 7.01(\mathrm{~d}, J=2.2 \mathrm{~Hz}, 1 \mathrm{H}), 6.93$ $6.87(\mathrm{~m}, 4 \mathrm{H}), 6.85-6.80(\mathrm{~m}, 3 \mathrm{H}), 5.11(\mathrm{~s}, 1 \mathrm{H}), 5.00(\mathrm{~s}, 2 \mathrm{H}), 4.44(\mathrm{~d}, J=11.7 \mathrm{~Hz}, 1 \mathrm{H})$ $4.35(\mathrm{~d}, J=11.3 \mathrm{~Hz}, 1 \mathrm{H}), 3.88(\mathrm{~s}, 3 \mathrm{H}), 3.80(\mathrm{~s}, 3 \mathrm{H}), 3.09-2.98(\mathrm{~m}, 2 \mathrm{H}), 2.89$ (dt, $J=$ $14.1,3.8 \mathrm{~Hz}, 2 \mathrm{H}), 2.84(\mathrm{~s}, 4 \mathrm{H}), 2.16(\mathrm{~m}, 1 \mathrm{H}), 1.90(\mathrm{~m}, 1 \mathrm{H}) \mathrm{ppm} ;{ }^{13} \mathrm{C}$ NMR $\left(100 \mathrm{MHz}, \mathrm{CDCl}_{3}\right): \delta=159.0(\mathrm{C}), 158.2(\mathrm{C}), 154.0(\mathrm{C}), 149.7(\mathrm{C}), 143.9(\mathrm{C}), 137.3(\mathrm{C})$ $137.1(\mathrm{C}), 134.8(\mathrm{C}), 134.0(\mathrm{C}), 132.7(\mathrm{C}), 131.9(\mathrm{CH}), 131.7(\mathrm{CH}), 130.6(\mathrm{C}), 129.2$ (C), $128.9(2 \times \mathrm{CH}), 128.8(\mathrm{CH}), 128.3(2 \times \mathrm{CH}), 127.5(\mathrm{CH}), 126.7(2 \times \mathrm{CH}), 125.1$ $(\mathrm{CH}), 121.9(\mathrm{CH}), 116.7(2 \times \mathrm{CH}), 114.4(\mathrm{CH}), 113.9(\mathrm{CH}), 113.1(\mathrm{CH}), 112.8(\mathrm{CH})$ $70.5\left(\mathrm{CH}_{2}\right), 56.1\left(\mathrm{CH}_{3}\right), 55.4\left(\mathrm{CH}_{3}\right), 50.8(\mathrm{CH}), 44.8\left(\mathrm{CH}_{2}\right), 37.0\left(\mathrm{CH}_{2}\right), 36.9\left(\mathrm{CH}_{2}\right)$, $32.1\left(2 \times \mathrm{CH}_{2}\right), 25.0\left(\mathrm{CH}_{2}\right) \mathrm{ppm}$; IR (film): v 2930, 1605, 1503, 1456, 1369, 1270, 1225 1165, 905; MS $\left(\mathrm{ESI}^{+}\right) \mathrm{m} / \mathrm{z}(\%): 707\left(\left[\mathrm{M}\left\{{ }^{37} \mathrm{Cl}\right\}+\mathrm{Na}\right]^{+}, 40 \%\right), 705\left(\left[\mathrm{M}\left\{{ }^{35} \mathrm{Cl}\right\}+\mathrm{Na}\right]^{+}\right.$ $100 \%)$

2,3,4,17,18,30-Hexahydro-22-Benzyloxy-13,27-dimethoxy-1,5-dithia-11-oxa-7,10etheno-12,16-metheno-19,23-methenobenzo[x]spiro[5,20]hexacosatridecaene (38): To a solution of dithiane $37(200 \mathrm{mg}, 0.29 \mathrm{mmol})$ in THF $(30 \mathrm{~mL})$ at $-78^{\circ} \mathrm{C}$ was added $n$ $\mathrm{BuLi}(2.44 \mathrm{M}$ in hexanes, $0.13 \mathrm{~mL}, 0.32 \mathrm{mmol}$ ) over $3 \mathrm{~min}$. The reaction mixture was warmed to $\mathrm{RT}$ and after $18 \mathrm{~h}$. sat. $\mathrm{NH}_{4} \mathrm{Cl}(10 \mathrm{~mL})$ and water $(5 \mathrm{~mL})$ were added. The aqueous phase was separated at extracted with $\mathrm{Et}_{2} \mathrm{O}(3 \times 20 \mathrm{~mL})$, then the combined organic phases were dried over $\mathrm{MgSO}_{4}$, concentrated in vacuo and purified by column chromatography (40-50 \% $\mathrm{Et}_{2} \mathrm{O} / \mathrm{petrol}$ ) to afford the title compound $\mathbf{3 8}$ as a white solid (92 mg, 48\%); MP 118-120 ${ }^{\circ} \mathrm{C}\left(\mathrm{Et}_{2} \mathrm{O} /\right.$ petrol); ${ }^{1} \mathrm{H} \mathrm{NMR}\left(400 \mathrm{MHz}, \mathrm{CDCl}_{3}\right): \delta=7.55$ (b $\mathrm{s}, 1 \mathrm{H}), 7.42(\mathrm{~d}, J=2.7 \mathrm{~Hz}, 1 \mathrm{H}), 7.37-7.23(\mathrm{~m}, 7 \mathrm{H}), 7.06(\mathrm{dd}, J=8.4,2.1 \mathrm{~Hz}, 1 \mathrm{H}), 6.94$ $(\mathrm{d}, J=8.6 \mathrm{~Hz}, 1 \mathrm{H}), 6.89-6.78(\mathrm{~m}, 4 \mathrm{H}), 6.80(\mathrm{~d}, J=1.9 \mathrm{~Hz}, 1 \mathrm{H}), 6.76(\mathrm{~d}, J=2.1 \mathrm{~Hz}$ $1 \mathrm{H}), 6.63(\mathrm{~d}, J=1.8 \mathrm{~Hz}, 1 \mathrm{H}), 4.95(\mathrm{~d}, J=12.2 \mathrm{~Hz}, 1 \mathrm{H}), 4.85(\mathrm{~d}, J=12.0 \mathrm{~Hz}, 1 \mathrm{H}), 3.91$ $(\mathrm{s}, 6 \mathrm{H}), 3.62(\mathrm{~d}, J=14.2 \mathrm{~Hz}, 1 \mathrm{H}), 3.52(\mathrm{~d}, J=14.0 \mathrm{~Hz}, 1 \mathrm{H}), 3.19-2.95(\mathrm{~m}, 3 \mathrm{H}), 2.72$ $2.55(\mathrm{~m}, 4 \mathrm{H}), 2.47(\mathrm{~m}, 1 \mathrm{H}), 1.98-1.87(\mathrm{~m}, 2 \mathrm{H}) \mathrm{ppm} ;{ }^{13} \mathrm{C} \mathrm{NMR}\left(100 \mathrm{MHz}, \mathrm{CDCl}_{3}\right): \delta=$ $157.6(\mathrm{C}), 154.6(\mathrm{C}), 153.1$ (C), $148.8(\mathrm{C}), 147.2(\mathrm{C}), 137.2(\mathrm{C}), 136.3(\mathrm{C}), 135.6(\mathrm{C})$ $134.8(\mathrm{C}), 134.6(\mathrm{C}), 133.4(\mathrm{CH}), 131.9(\mathrm{C}), 131.7(\mathrm{CH}), 130.8(\mathrm{C}), 128.4(2 \times \mathrm{CH})$ $127.6(\mathrm{CH}), 127.2(\mathrm{CH}), 127.2(2 \times \mathrm{CH}), 127.1(2 \times \mathrm{CH}), 122.0(\mathrm{CH}), 117.5(\mathrm{CH})$ $116.1(\mathrm{CH}), 113.8(2 \times \mathrm{CH}), 113.0(\mathrm{CH}), 112.1(2 \times \mathrm{CH}), 71.1\left(\mathrm{CH}_{2}\right), 59.7(\mathrm{C}), 56.3$ $\left(\mathrm{CH}_{3}\right), 55.3\left(\mathrm{CH}_{3}\right), 49.5\left(\mathrm{CH}_{2}\right), 34.0\left(\mathrm{CH}_{2}\right), 31.8\left(\mathrm{CH}_{2}\right), 28.2\left(\mathrm{CH}_{2}\right), 27.5\left(\mathrm{CH}_{2}\right), 25.2$ $\left(\mathrm{CH}_{2}\right)$ ppm; IR (solid): v 2925, 2852, 1605, 1513, 1496, 1463, 1270, 1230, 1166, 1128 MS $\left(\mathrm{ESI}^{+}\right) \mathrm{m} / \mathrm{z}(\%): 669\left([\mathrm{M}+\mathrm{Na}]^{+}, 100 \%\right)$; HRMS $\left(\mathrm{ESI}^{+}\right)$: calcd for $\mathrm{C}_{40} \mathrm{H}_{38} \mathrm{NaO}_{4} \mathrm{~S}_{2}$ $[\mathrm{M}+\mathrm{Na}]^{+} 669.2104$; found: 669.2103

1,2,13,14-Tetrahydro-9,23-dimethoxy-18-hydroxy-3,6-etheno-8,12-metheno-15,19metheno-7-oxabenzo[t]cycloeicosadecaene (39): To a solution of $\mathrm{NiCl}_{2} .6 \mathrm{H}_{2} \mathrm{O}(310 \mathrm{mg}$, $1.28 \mathrm{mmol})$ in DMF $(1 \mathrm{~mL})$ was added sequentially a solution of dithiane $38(0.052 \mathrm{~g}$, $0.08 \mathrm{mmol})$ in THF $(2 \mathrm{~mL})$ and $\mathrm{NaBH}_{4}(96 \mathrm{mg}, 2.56 \mathrm{mmol})$. After $18 \mathrm{~h}$ the mixture filtered through Celite ${ }^{\odot}$, concentrated in vacuo and partitioned between water $(10 \mathrm{~mL})$ and $\mathrm{Et}_{2} \mathrm{O}(15 \mathrm{~mL})$. The aqueous phase was separated and extracted with $\mathrm{Et}_{2} \mathrm{O}(2 \times 15$ $\mathrm{mL}$ ), then the combined organic phases were dried over $\mathrm{MgSO}_{4}$, concentrated in vacuo and purified by column chromatography $\left(30 \% \mathrm{Et}_{2} \mathrm{O} /\right.$ petrol) to give a white solid ( 25 $\mathrm{mg})$. That material was dissolved in EtOH $(3 \mathrm{~mL})$ and DCM $(3 \mathrm{~mL})$ and $10 \%$ Pd-C (10 $\mathrm{mg}$ ) added. The flask was evacuated and purged with argon three times then evacuated and purged with hydrogen twice. After $6 \mathrm{~h}$ the reaction mixture was filtered through Celite $^{\odot}$, concentrated in vacuo and purified by column chromatography (50-70\% $\mathrm{Et}_{2} \mathrm{O} / \mathrm{h}$ exane) to afford the title compound 39 as a white solid $(15.8 \mathrm{mg}, 74 \%)$; MP 85 $87{ }^{\circ} \mathrm{C}\left(\mathrm{Et}_{2} \mathrm{O} /\right.$ petrol $) ;{ }^{1} \mathrm{H}$ NMR $\left(400 \mathrm{MHz}, \mathrm{CDCl}_{3}\right): \delta=7.10(\mathrm{~d}, J=2.6 \mathrm{~Hz}, 1 \mathrm{H}), 7.05(\mathrm{~d}$, $J=8.6 \mathrm{~Hz}, 2 \mathrm{H}), 6.88(\mathrm{dd}, J=8.6,2.7 \mathrm{~Hz}, 2 \mathrm{H}), 6.84(\mathrm{~d}, J=7.3 \mathrm{~Hz}, 1 \mathrm{H}), 6.80(\mathrm{~d} J=8.1$ $\mathrm{Hz}, 1 \mathrm{H}), 6.77-6.70(\mathrm{~m}, 3 \mathrm{H}), 6.62(\mathrm{~d}, J=1.8 \mathrm{~Hz}, 1 \mathrm{H}), 6.33(\mathrm{br} \mathrm{s}, 1 \mathrm{H}), 6.29(\mathrm{~d}, J=2.0$ $\mathrm{Hz}, 1 \mathrm{H}), 4.62(\mathrm{~s}, 1 \mathrm{H}), 3.92(\mathrm{~s}, 6 \mathrm{H}), 3.18(\mathrm{br} \mathrm{dd}, J=13.5,3.9 \mathrm{~Hz}, 1 \mathrm{H}), 3.07-2.85(\mathrm{~m}$ $5 \mathrm{H}), 2.49-2.73(\mathrm{~m}, 2 \mathrm{H}) \mathrm{ppm} ;{ }^{13} \mathrm{C} \mathrm{NMR}\left(100 \mathrm{MHz}, \mathrm{CDCl}_{3}\right): \delta=159.7(\mathrm{C}), 153.7(\mathrm{C})$ 150.1 (C), 149.8 (C), 147.0 (C), 141.0 (C), 137.6 (C), 136.3 (C), 134.8 (C), $133.1(2 \times$ $\mathrm{CH}), 130.4(\mathrm{CH}), 129.9(\mathrm{CH}), 129.3(\mathrm{CH}), 129.2(\mathrm{C}), 128.5(\mathrm{CH}), 126.8(\mathrm{C}), 121.5$ $(\mathrm{CH}), 121.0(\mathrm{CH}), 115.4(\mathrm{CH}), 114.9(\mathrm{CH}), 114.8(\mathrm{CH}), 113.0(\mathrm{CH}), 111.6(\mathrm{CH}), 56.2$ $\left(\mathrm{CH}_{3}\right), 55.3\left(\mathrm{CH}_{3}\right), 37.8\left(\mathrm{CH}_{2}\right), 35.2\left(\mathrm{CH}_{2}\right), 34.9\left(\mathrm{CH}_{2}\right), 33.4\left(\mathrm{CH}_{2}\right) \mathrm{ppm}$; IR (solid): v

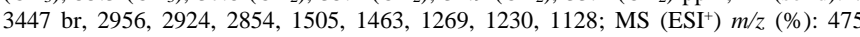
$\left([\mathrm{M}+\mathrm{Na}]^{+}, 100 \%\right)$; HRMS $\left(\mathrm{ESI}^{+}\right)$: calcd for $\mathrm{C}_{30} \mathrm{H}_{28} \mathrm{NaO}_{4}[\mathrm{M}+\mathrm{Na}]^{+} 475.1880$; found 475.1879 .

1,2,13,14-Tetrahydro-9,18,23-trihydroxy-3,6-etheno-8,12-metheno-15,19-metheno-7oxabenzo[t]cycloeicosadecaene (5): To a stirred solution of the macrocyclic bisbibenzyl $39(150 \mathrm{mg}, 0.33 \mathrm{mmol})$ in DCM $(33 \mathrm{~mL})$ was added boron tribromide (1 M solution in DCM, $3.31 \mathrm{~mL}, 3.31 \mathrm{mmol})$. After $5 \mathrm{~h}$, water $(10 \mathrm{~mL})$ was added then the aqueous phase was separated and extracted with $\mathrm{Et}_{2} \mathrm{O}(3 \times 30 \mathrm{~mL})$. The combined organic phases were dried over $\mathrm{MgSO}_{4}$, concentrated in vacuo and purified by column chromatography ( $40 \% \mathrm{Et}_{2} \mathrm{O} / \mathrm{petrol}$ ) to afford the title compound $\mathbf{5}$ as a white solid (130 mg, 92\%); MP $154-155{ }^{\circ} \mathrm{C}\left(\mathrm{Et}_{2} \mathrm{O} /\right.$ hexane $^{\prime}{ }^{1} \mathrm{H}$ NMR $\left(400 \mathrm{MHz}, \mathrm{CD}_{3} \mathrm{OD}\right): \delta=6.93(\mathrm{~d}, J$ $=2.6 \mathrm{~Hz}, 1 \mathrm{H}), 6.89(\mathrm{dd}, J=8.4,2.1 \mathrm{~Hz}, 1 \mathrm{H}), 6.80(\mathrm{~d}, J=8.4 \mathrm{~Hz}, 1 \mathrm{H}), 6.74(\mathrm{~d}, J=1.2$ $\mathrm{Hz}, 1 \mathrm{H}), 6.69(\mathrm{~d}, J=8.1 \mathrm{~Hz}, 1 \mathrm{H}), 6.66-6.62(\mathrm{~m}, 2 \mathrm{H}), 6.61-6.56(\mathrm{~m}, 3 \mathrm{H}), 6.51(\mathrm{~d}, J=$ $2.2 \mathrm{~Hz}, 1 \mathrm{H}), 6.32(\mathrm{br} \mathrm{s}, 1 \mathrm{H}), 6.22(\mathrm{~d}, J=2.0 \mathrm{~Hz}, 1 \mathrm{H}), 3.04(\mathrm{br} \mathrm{dd}, J=12.9,4.8 \mathrm{~Hz}, 1 \mathrm{H})$, 2.96-2.65 (m, 6H), $2.43(\mathrm{~m}, 1 \mathrm{H}) \mathrm{ppm} ;{ }^{13} \mathrm{C} \mathrm{NMR}\left(100 \mathrm{MHz}, \mathrm{CD}_{3} \mathrm{OD}\right): \delta=157.9(\mathrm{C})$ $155.6(\mathrm{C}), 152.8$ (C), $149.6(\mathrm{C}), 145.5(\mathrm{C}), 143.4$ (C), 139.8 (C), $136.6(\mathrm{C}), 135.0$ (C), $133.4(\mathrm{CH}), 132.8(\mathrm{CH}), 132.4(\mathrm{C}), 130.7(\mathrm{CH}), 129.7(\mathrm{C}), 128.5(\mathrm{CH}), 122.7(\mathrm{br}, 2 \times$ $\mathrm{CH}), 121.6(\mathrm{br}, 2 \times \mathrm{CH}), 116.9(\mathrm{CH}), 116.8(\mathrm{CH}), 116.8(\mathrm{CH}), 116.3(\mathrm{CH}), 114.4(\mathrm{CH})$ 
$38.8\left(\mathrm{CH}_{2}\right), 37.1\left(\mathrm{CH}_{2}\right), 36.0\left(\mathrm{CH}_{2}\right), 34.5$ $\left(\mathrm{CH}_{2}\right)$ ppm; IR (solid): v 3363 br, 2954, 2923, 2853, 1505, 1445, 1222, 1163; MS $\left(\mathrm{ESI}^{-}\right) \mathrm{m} / \mathrm{z}(\%): 447\left([\mathrm{M}+\mathrm{Na}]^{+}, 100 \%\right)$; HRMS $\left(\mathrm{ESI}^{+}\right)$: calcd for $\mathrm{C}_{28} \mathrm{H}_{25} \mathrm{O}_{4}$ $[\mathrm{M}+\mathrm{H}]^{+}$425.1747; found: 425.1743; Xray: CCDC 1438902 , see insert.

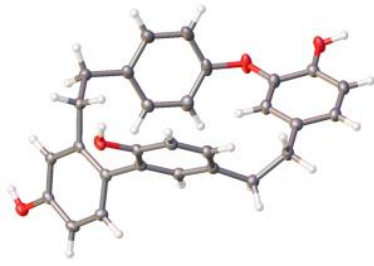

\section{Acknowledgements}

We gratefully acknowledge the funding provided by Umm Al-Qura University, Saudi Arabia and EPSRC via equipment grant EP/K039466/1. We also thank Dr Mark Light for the X-ray analysis and Dr Paul Murgues for advice and guidance (to FAA).

\section{References}

[1] a) Y. Asakawa, Phytochem. 2004, 65, 623-669; b) G. M. Keserü, M. Nógrádi, Nat. Prod. Rep. 1995, 12, $69-75$; c) H. D. Zinsmeister, H. Becker, T. Eicher Angew. Chem. Int. Ed. Engl. 1991, 30, 130-147.

[2] D. C. Harrowven, S. L. Kostiuk, Nat. Prod. Rep. 2012, 29, 223-242.

[3] a) Y. S. Kosenkova, M. P. Polovinka, N. I. Komarova, D. V. Korchagina, N. Y Kurochkina, V. A. Cheremushkina, N. F. Salakhutdinov, Chem. Nat. Compd. 2007, 43, 712-713; b) Y. S. Kosenkova, M. P. Polovinka, N. I. Komarova, D. V. Korchagina, S. V. Morozov, A. I. Vyalkov, N. Y. Kurochkina, V. A., Cheremushkina, N. F. Salakhutdinov, Chem. Nat. Compd. 2008, 44, 564-568.

[4] a) L. Wang, L.-N. Wang, Y. Zhao, H.-X. Lou, A.-X. Cheng, Nat. Prod. Res. 2013, 27, 274-276; b) C.-F. Xie, J.-B. Qu, X.-Z. Wu, N. Liu, M. Ji, H.X. Lou, Nat. Prod. Res. 2010, 24, 515-520; c) L. Harinantenaina, Y. Asakawa, Chem. Pharm. Bull. 2004, 52, 1382-1384; d) A. Bardon, N. Kamiya, M. Toyota, S Takaoka, Y. Asakawa, Phytochem. 1999, 52, 1323-1329; e) H. Anton, R. Schoeneborn, R. Mues, Phytochem. 1999, 52, 1639-1645; f) C.-L. Wu, H.-R. Lin, Phytochem. 1997, 44, 101-105; g) M. Toyota, T. Yoshida, J. Matsunami, Y Asakawa, Phytochem. 1997, 44, 293-298; h) T. Yoshida, T. Hashimoto, S. Takaoka, Y. Kan, M. Tori, Y. Asakawa, J. M. Pezzuto, T. Pengsuparp, G. A. Cordell, Tetrahedron 1996, 52, 14487-14500; i) T. Hashimoto, T. Yoshida, Y Kan, S. Takaoka, M. Tori, Y. Asakawa, Tetrahedron Lett. 1994, 35, 909-910; j) S. Kunz, H. Becker, Phytochem. 1992, 31, 3981-3984; k) Y. Asakawa, K Okada, G. W. Perold, Phytochem. 1988, 27, 161-164; 1) M. Toyota, F Nagashima, Y. Asakawa, Phytochem. 1988, 27, 2603-2608; m) Y. Asakawa, M. Tori, K. Takikawa, H. G. Krishnamurty, S. K. Kar, Phytochem. 1987, 26, 18111816 ; n) Y. Asakawa, M. Toyota, R. Matsuda, K. Takikawa, T. Takemoto, Phytochem. 1983, 22, 1413-1416; o) Y. Asakawa, R. Matsuda, Phytochem. 1982, 21, 2143-2144.

[5] a) Y. Asakawa, Prog. Chem. Org. Nat. Prod. 1995, 65, 335-351; b) M. Toyota, T. Kinugawa, Y. Asakawa, Phytochem., 1994, 37, 859-862; c) M. Toyota, M Tori, K. Takikawa, Y. Shiobara, M. Kodama, Y. Asakawa, Tetrahedron Lett. 1985, 26, 6097-6100.

[6] M. Toyota; T. Yoshida; Y. Kan; S. Takaoka; Y. Asakawa, Tetrahedron Lett. 1996, 37, 4745-4748

[7] Qu, C. Xie, H. Guo, W. Yu, H. Lou, Phytochem. 2007, 68, 1767-1774.

[8] a) A. Gottsegen, M. Nógrádi, B. Vermes, M. Kajtár-Peredy; É. Bihátsi-Karsai, J. Chem. Soc., Perkin Trans. 1, 1990, 315-320; b) A. Gottsegen, M. Nógrádi, B. Vermes, M. Kajtár-Peredy, É. Bihátsi-Karsai, Tetrahedron Lett. 1988, 29, 50395040 .

[9] a) H. Sawada, K. Onoda, D. Morita, E. Ishitsubo, K. Matsuno, H. Tokiwa, T Kuroda, H. Miyachi, Bioorg. Med. Chem. Lett. 2013, 23, 6563-6568; b) A Speicher, M. Groh, M. Hennrich, A.-M. Huynh, Eur. J. Org. Chem. 2010 6760-6778; c) T. Eicher, S. Fey, W. Puhl, E. Büchel, A. Speicher, Eur. J. Org. Chem. 1998, 877-888.

[10] S. L. Kostiuk, T. Woodcock, L. F. Dudin, P. D. Howes, D. C. Harrowven, Chem Eur. J. 2011, 17, 10906-10915.
[11] a) H. Hioki, N. Shima, K. Kawaguchi, K. Harada, M. Kubo, T Esumi, T. Nishimaki-Mogami, J.-i. Sawada, T. Hashimoto, Y. Asakawa, Y. Fukuyama, Bioorg. Med. Chem. Lett. 2009, 19, 738-741; b) K. Dodo, A. Aoyama, T. Noguchi-Yachide, M. Makishima, H. Miyachi, Y. Hashimoto, Bioorg. Med. Chem. 2008, 16, 4272-4285.

[12] D. C. Harrowven, T. Woodcock, P. D. Howes, Angew. Chem. Int. Ed. 2005, 44 3899-3901.

[13] K. Harada, K. Makino, N. Shima, H. Okuyama, T. Esumi, M. Kubo, H. Hioki, Y. Asakawa, Y. Fukuyama, Tetrahedron 2013, 69, 6959-6968.

[14] M. Iwashita, S. Fujii, S. Ito, T. Hirano, H. Kagechika, Tetrahedron 2011, 67, 6073-6082.

[15] C. F. Xie, J. B. Qu, X. Z. Wu, N. Liu, M. Ji, H. X. Lou, Nat. Prod. Res. 2010, 24, 515-520.

[16] K. Fujii, D. Morita, K. Onoda, T. Kuroda, H. Miyachi, Bioorg. Med. Chem. Lett. 2016, 26, 2324-2327

[17] a) X. Xue, D. F. Sun, C. C. Sun, H. P. Liu, B. Yue, C. R. Zhao, H. X. Lou, X. J. Qu, Lung Cancer, 2012, 76, 300-308; b) W.-J. Huang, C.-L. Wu, C.-W. Lin, L.L. Chi, P.-Y. Chen, C.-J. Chiu, C.-Y. Huang, C.-N. Chen, Cancer Lett., 2010 , 291, 108-119; c) Y. Asakawa, M. Toyota, Z. Taira, T. Takemoto, M. Kido, J. Org. Chem. 1983, 48, 2164-2167.

[18] a) K. Fujii, D. Morita, K. Onoda, T. Kuroda, H. Miyachi, Bioorg. Med. Chem. Lett. 2016, 26, 2324-2327; b) H. Sawada, K. Onoda, D. Morita, E. Ishitsubo, K. Matsuno, H. Tokiwa, T. Kuroda, H. Miyachi, Bioorg. Med. Chem. Lett. 2013, 23 , 6563-6568; c) H. Sawada, M. Okazaki, D. Morita, T. Kuroda, K. Matsuno, Y. Hashimoto, H. Miyachi, Bioorg. Med. Chem. Lett. 2012, 22, 7444-7447; d) L. Harinantenaina, D. N. Quang, N. Takeshi, T. Hashimoto, C. Kohchi, G.-I. Soma, Y. Asakawa, J. Nat. Prod. 2005, 68, 1779-1781.

[19] H. Takiguchi, K. Ohmori, K. Suzuki, Angew. Chem. Int. Ed. 2013, 52, 10472 10476.

[20] a) P. Zhao, C. M. Beaudry, Org. Lett. 2013, 15, 402-405; b) J. H. Dam, PhD Thesis, Technical University of Denmark, 2009.

[21] K. Makino, K. Harada, M. Kubo, H. Hioki, Y. Fukuyama, Nat. Prod. Commun. 2013, 8, 915-918.

[22] a) P. C. B. Page, M. B. Vanniel, J. C. Prodger, Tetrahedron, 1989, 45, 7643 7677; b) D. Seebach, Angew. Chem., Int. Ed. 1979, 239-258; c) B.-T. Gröbel, D Seebach, Synthesis, 1977, 357-402; d) D. Seebach, E. J. Corey, J. Org. Chem., 1975, 40, 231-237; e) L. Field, Synthesis 1972, 101-131; f) D. Seebach, Synthesis, 1969, 17-36.

[23] A. B. Smith III, C. M. Adams, Acc. Chem. Res. 2004, 37, 365-377; M. Yus, C. $\mathrm{Na}$ jera, F. Foubelo, Tetrahedron 2003, 59, 6147-6212.

[24] For an example of the use of the Corey-Seebach reaction for construction of an acyclic bisbibenzyl see: P. Várnai, M. Nógrádi, ACH - Models Chem. 1999, 136, $275-286$.

[25] N. Finch, C. W. Gemenden, J. Org. Chem. 1979, 44, 2804-2805.

[26] a) A. Huth, I. Beetz, I. Schumann, Tetrahedron 1989, 45, 6679-6682; b) T. Ohe, N. Miyaura, A. Suzuki, Synlett 1990, 221-223; c) J.-M. Fu, V. Snieckus, Tetrahedron Lett. 1990, 31, 1665-1668; d) T. Ohe, N. Miyaura, A. Suzuki, J. Org. Chem. 1993, 58, 2201-2208.

[27] a) Q. Yao, E. P. Kinney, Z. Yang, J. Org. Chem. 2003, 68, 7528-7531; b) R. F. Heck, J. P. Nolley Jr., J. Org. Chem. 1972, 37, 2320 - 2322.

[28] As detailed in the experimental section, use of thionyl chloride gave a higher yield in this series (67\%) but proved less useful in the mimetic synthesis of $\mathbf{5}$.

[29] a) N. Gaggero, D. C. M. Albanese, N. Donatella, Tetrahedron 2014, 70, 8744 8749; b). G. Back, D. L. Baron, K. Yang, K. J. Org. Chem. 1993, 58, $2407-$ 2413 ; c) S. S. Zaman, P. Sarmah, N. C. Barua, R. P. Sharma, Chem. Ind. 1989, 806-807.

[30] a) M. Zhang, G. E. Jagdmann Jr., M. Van Zandt, P. Beckett, H. Schroeter, Tetrahedron Asymm. 2013, 24, 362-373; b) S. Li, M. L. Szalai, R. M. Kevwitch, D. V. McGrath, J. Am. Chem. Soc. 2003, 125, 10516-10517.

[31] H. E. Ungnade, J. Am. Chem. Soc. 1941, 63, 2091-2093.

Received: ((will be filled in by the editorial staff))

Revised: ((will be filled in by the editorial staff)) Published online: ((will be filled in by the editorial staff)) 
Entry for the Table of Contents (Please choose one layout only)

Layout 1:

Catch Phrase

Author(s), Corresponding

Author $(s) *$............ Page - Page

\section{Title Text}

Layout 2:

Total Synthesis

Faisal A. Almalki, David C.

Harrowven* ......Page - Page

\section{A Corey-Seebach \\ Macrocyclisation Strategy \\ for the Synthesis of \\ Riccardin $C$ and an \\ Unnatural Macrocyclic \\ Bisbibenzyl Analogue.}

Text for Table of Contents, max. 450 characters.
((The TOC Graphic should not exceed the size of this area))

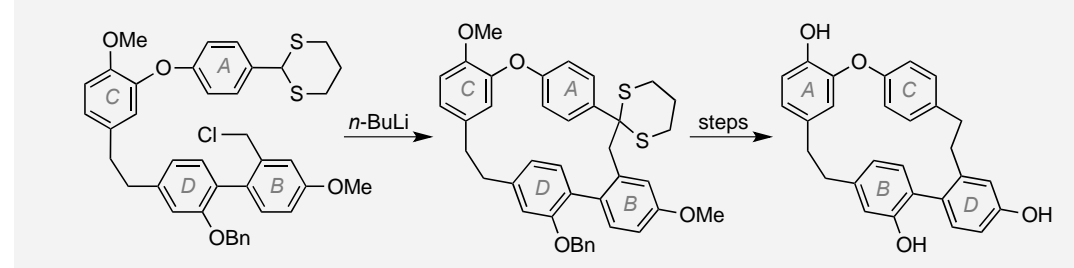

A total synthesis of riccardin $\mathrm{C}$ has been achieved featuring the use of a Corey-Seebach reaction to effect macrocyclisation. Through intersection with a late stage intermediate, formal syntheses of cavicularin and asterelin A have also been realised. More importantly, the approach has been used mimetically to prepare a new unnatural macrocyclic bisbibenzyl core. 